\title{
Facet-dependent Photocatalysis of Nanosize Semiconductive Metal Oxides and Progress of their Characterization
}

\author{
Yung-Kang Peng and S. C. Edman Tsang*
}

Wolfson Catalysis Centre, Department of Chemistry, University of Oxford, Oxford OX1 3QR, UK E-mail: edman.tsang@chem.ox.ac.uk

\begin{abstract}
Semiconductive metal oxides are of great importance in environmental remediation and electronics because of their ability to generate charge carriers when excited with appropriate light energy. The electronic structure, light absorption and charge transport properties of the metal oxides have made possible their applications as photocatalysts. Recently, facet-engineering by morphology control has been intensively studied as an efficient approach to further enhance their photocatalytic performance. However, various processing steps and post-treatments used during the preparation of facet-engineered particles may generate different surface active sites which may affect their photocatalysis. Moreover, many traditional techniques (PL, EPR, XPS and Raman) used for materials characterization (oxygen vacancy, hydroxyl group, cation...etc.) are not truly surface specific but the analyses range from top few layers to bulk. Accordingly, they can only provide very limited information on the chemical states of the surface active features and distributions among facets, causing difficulty to unambiguously correlate facet-dependent results with activity. As a result, this always leads to different interpretations amongst researchers during the past decades. In this article, we will review on the controversies generated among researchers, when they correlated the performance of two most popular photocatalysts, $\mathrm{ZnO}$ and $\mathrm{TiO}_{2}$ with their facet activities based on characterization from the traditional techniques. As there are shortcomings of these techniques in producing truly facet-dependent features, some results can be misleading and with no cross-literature comparison. This review is also focussed on the new capability of probe-molecule-assisted NMR which allows a genuine differentiation of surface active sites from various facets. This surface-fingerprint
\end{abstract}


technique has been demonstrated to provide both qualitative (chemical shift) and quantitative (peak intensity) information on the concentration and distribution of truly surface features. In light of the new technique this article will revisit the facet-dependent photocatalytic properties and shed light on these issues.

\section{Introduction}

Environmental pollution becomes one of the most severe issues we are currently facing on a global scale. The release of toxic chemicals and industrial wastes into air and water has resulted in the up-rises of pollution-related diseases and climatic changes. Photocatalysis using inexhaustibly irradiation of the Sun has shown its potential as an environmentally sustainable and economically viable way to address those concerns during the past decades. ${ }^{[1,2]}$ The term 'photocatalysis' consists of the combination of photochemistry and catalysis implying that light and a catalyst are necessary to initiate or to accelerate a redox reaction. In most cases, heterogeneous photocatalysis involving photoreactions at the catalyst surface refers to semiconductor photocatalysis. Nowadays, semiconductive transition metal oxide materials with well-defined sizes, structures, compositions and shapes have been extensively developed as photocatalysts for various reactions. ${ }^{[3-5]}$ Potential applications include pollutant photodegradation, ${ }^{[6]}$ photocatalytic water splitting, ${ }^{[7]} \mathrm{CO}_{2}$ photoreduction, ${ }^{[8]}$ artificial photosynthesis, ${ }^{[9]}$ alcohol photoreforming, ${ }^{[10]}$ and photoelectrochemical conversion. ${ }^{[1]} \mathrm{Up}$ to date, in addition to traditional semiconductive photocatalysts such as $\mathrm{ZnO},{ }^{[12,13]} \mathrm{TiO}_{2},{ }^{[14,15]} \mathrm{WO}_{3},{ }^{[16,17]} \mathrm{CdSe}^{[18]}$ and $\mathrm{CdS},{ }^{[19]}$ a wide variety of novel semiconductive materials have been explored as photocatalysts such as $\mathrm{MoS}_{2},{ }^{[20]} \mathrm{C}_{3} \mathrm{~N}_{4},{ }^{[21]}$ and metal-organic framework $(\mathrm{MOF})^{[22]}$ based materials, etc. However, $\mathrm{ZnO}$ and $\mathrm{TiO}_{2}$ remain to be the most popular materials and are regarded as a benchmark in the field of photocatalysis.

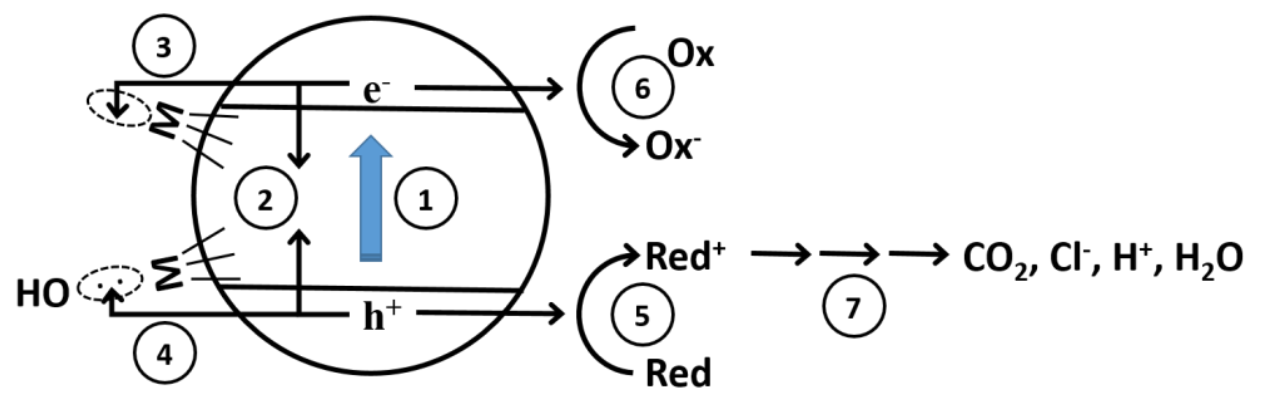

Figure 1. Seven primary steps in the mechanism of photocatalysis. Reproduced with permission. ${ }^{[23]}$ Copyright 1995, American Chemical Society. 
In general, there are seven basic steps involved in the mechanism of semiconductor photocatalysis ${ }^{[23]}$ (Figure 1): (1) formation of charge carriers by photon absorption; (2) charge carrier recombination; (3) trapping of a conduction-band electron at a surface cation site (i.e. $\mathrm{M}^{\mathrm{n}+}$ ) to yield $\mathrm{M}^{(\mathrm{n}-1)+}$; (4) trapping of a valence-band hole at a surficial M-OH group; (5) initiation of an oxidative pathway by a valence-band hole; (6) initiation of a reductive pathway by a conduction-band electron; and (7) further thermal (e.g., hydrolysis or reactions with active oxygen species) and photocatalytic reactions to yield mineralization products. Fundamental studies concerning those very fast photocatalytic processes inside and at the surface of the semiconductor, are not carried out very often. However, the knowledge of these processes is of utmost importance for the understanding of the photocatalytic reaction mechanism and thus for a better design of photocatalytic systems. Figure 2 provides an overview of the photo-induced events inside and on the surface of a $\mathrm{TiO}_{2}$ photocatalyst in the time scale region from femtoseconds to microseconds. ${ }^{[3]}$ Generally, the efficiency of photocatalytic processes is rather small that most of the photogenerated $\mathrm{e}^{-} / \mathrm{h}^{+}$pairs $(\sim 90 \%)$ recombine rapidly after excitation and therefore low photonic efficiency $(<10 \%)$ for most semiconductor-based photocatalytic reactions. ${ }^{[23]}$ Since this recombination process is the major disadvantage in semiconductor photocatalysis, many strategies have been adopted during the past to improve the photonic efficiency (inhibit recombination process). 


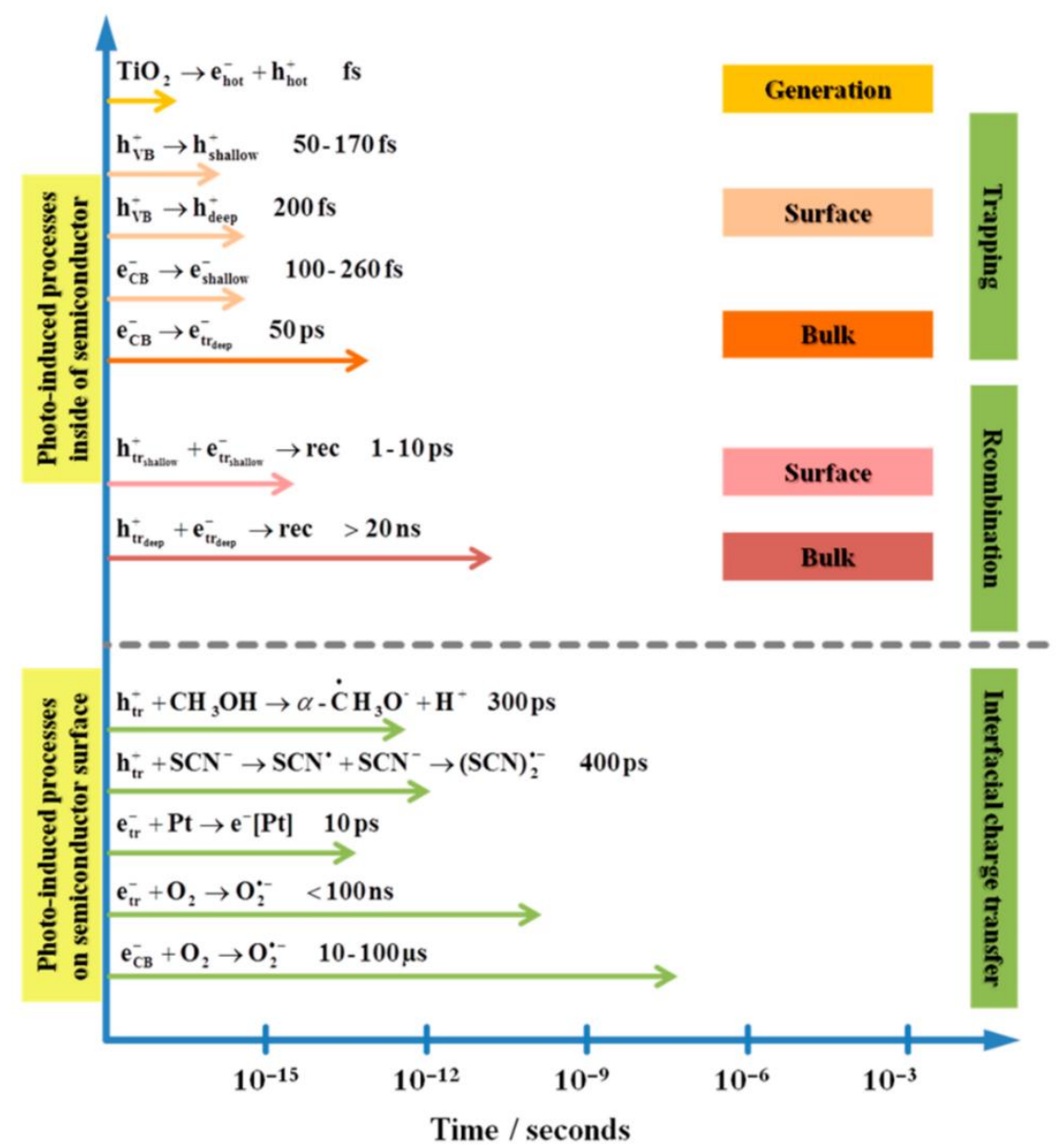

Figure 2. Photoinduced reactions in $\mathrm{TiO}_{2}$ photocatalysis and corresponding time scales. Reproduced with permission. ${ }^{[3]}$ Copyright 2014, American Chemical Society.

\section{Strategies to improve photocatalytic efficiency}

Comparing to the time scale of charge recombination in bulk (nano second, Figure 2), the photogenerated $\mathrm{e}^{-} / \mathrm{h}^{+}$can be trapped by surface states forming $\mathrm{e}_{\text {shallow }}{ }^{-} / \mathrm{h}_{\text {shallow }}{ }^{+} / \mathrm{h}_{\text {deep }}{ }^{+}$in a much shorter time scale (femto second) right after the generation of $\mathrm{e}^{-} / \mathrm{h}^{+}$pairs. ${ }^{[24]}$ Accordingly, the increase of surface states can effectively extract photogenerated $\mathrm{e}^{-} / \mathrm{h}^{+}$to catalyst surface for later redox reaction and avoid their recombination in bulk. Therefore, the shrinkage of particle size has been pursued as a strategy to boost the number of trapped sites (catalytic sites) on catalyst surface for a better photocatalytic activity. On the other hand, once the size of a semiconductor particle falls below the critical radius, the charge carriers begin to behave quantum mechanically restricted and the charge confinement leads to a series of discrete electronic states. This results in an increase in the effective band gap and a shift of the band edges. It is thus possible to enhance the redox potential of the valence-band holes and the conduction band electrons by decreasing the size of the semiconductor particles. ${ }^{[25]}$ Later, cation/anion doping has also been adopted to further counter the recombination problem by enhancing the charge separation as the dopants may more efficiently trap 
electrons/holes. ${ }^{[1-4]}$ However, as shown in Figure 2, the recombination rate of surface $\mathrm{e}_{\text {shallow }}{ }^{-}$and $\mathrm{h}_{\text {shallow }}{ }^{+}$ (pico second) is still at least ten times faster than the interfacial charge transfer (i.e. steps (5) and (6) in Figure 1), which is the rate limiting step in heterogeneous photocatalysis. According to Fogler, ${ }^{[26]}$ this interfacial charge transfer can be divided into following five independent steps as shown in Figure 3: (1) Mass transfer of the reactant(s) (e.g. A) in the bulk fluid/gas phase to the surface. (2) Adsorption of the reactant(s) onto the photon activated surface. (3) Photocatalysis reaction for the adsorbed phase on the surface (e.g. A/B). (4) Desorption of the intermediate(s) (e.g. B) from the surface. (5) Mass transfer of the intermediate(s) (e.g. B) from the interface region to the bulk fluid/gas.

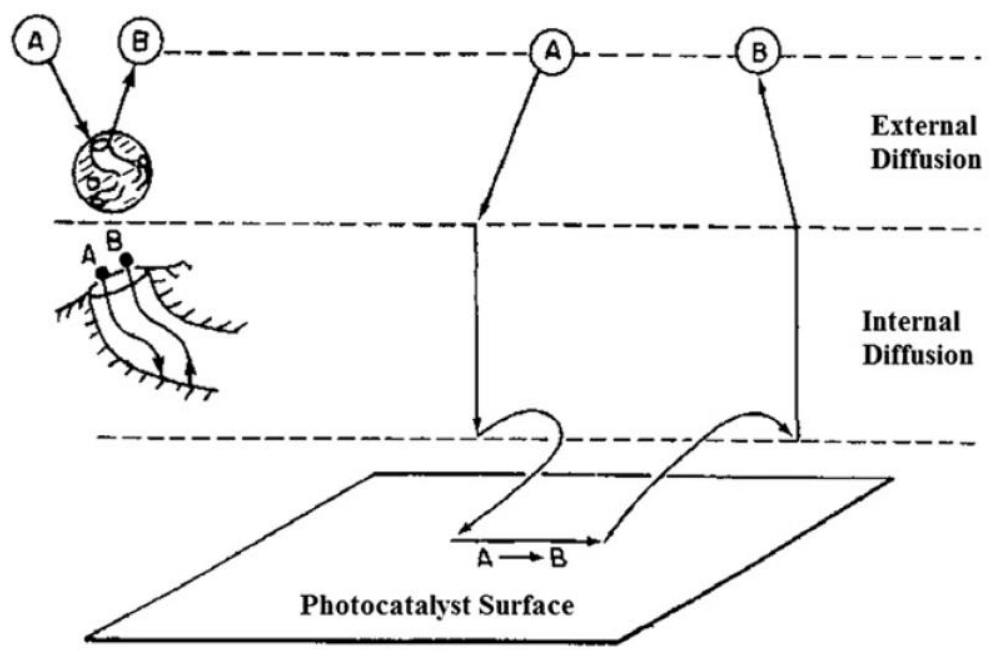

Figure 3. Adsorption/desorption steps in heterogeneous catalytic reaction. Reproduced with permission. ${ }^{[26]}$ Copyright 1991, Prentice-Hall PTR Inc.

In terms of rate determination, the overall rate of photocatalytic reaction is equal to the slowest step of all the elementary steps of the reaction. As the mass transfer steps (step 1 and 5) are usually very fast compared with the reaction steps (step 2, 3 and 4), the molecules in the immediate vicinity of the active sites are indistinguishable from those in the bulk fluid/gas phase. In this scenario, the mass transfer steps are unlikely to be rate limiting and do not affect the overall rate of photocatalytic reaction. Vinodgopal and Kamat first reported the dependence of the photodegradation rate of the organic molecules on surface coverage of the photocatalysts used. ${ }^{[27]}$ This outlines the importance of the adsorption-reaction-desorption interactions between molecules and surface of the catalyst during the reaction (step 2, 3 and 4). Recently, the surface/facet-tailoring for photocatalytic materials has indeed been proved an efficient strategy to further enhance their photocatalytic performance. ${ }^{[5]}$ 


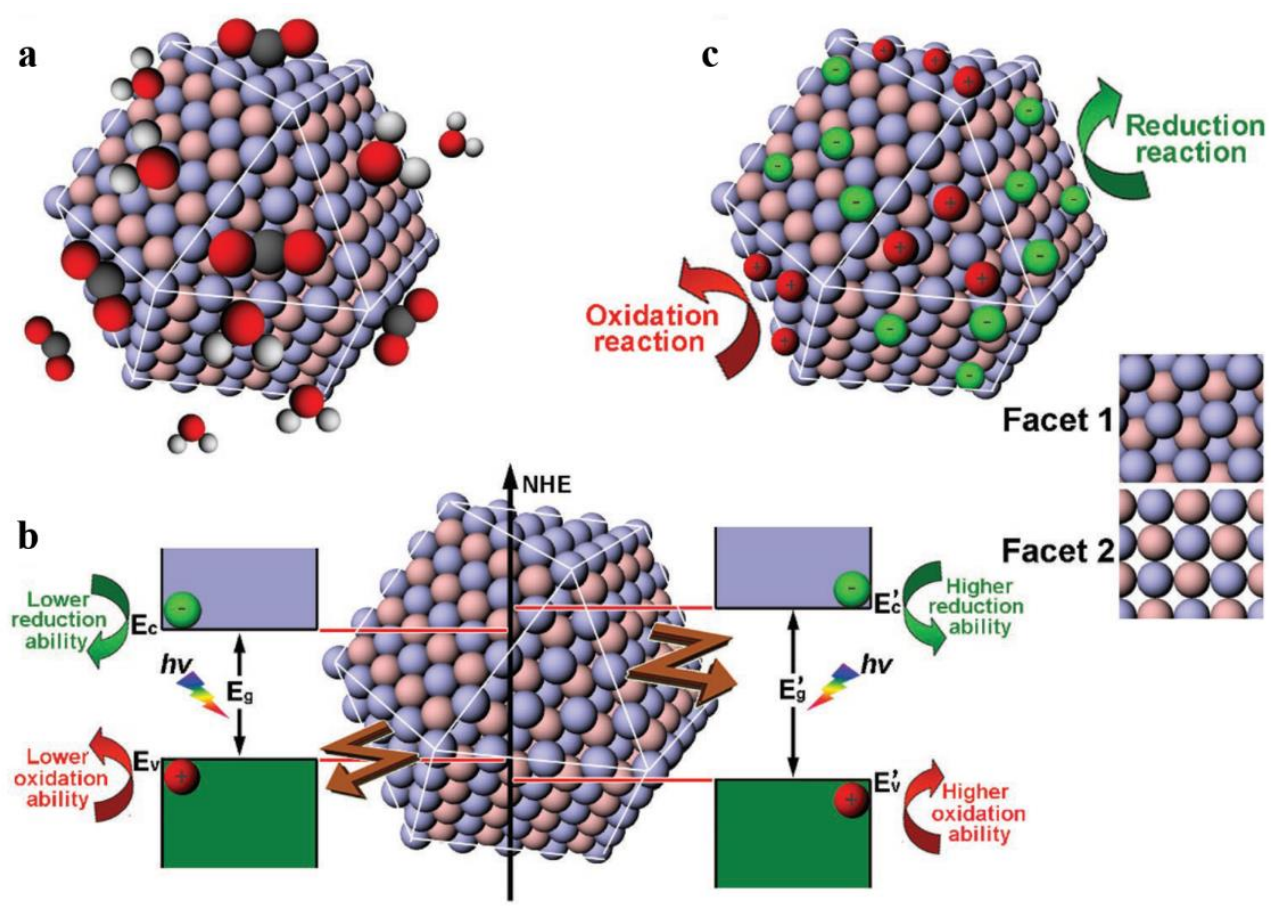

Figure 4. Schematics illustrating the important roles of facets in the surface design of photocatalytic materials. a) Adsorption and activation of reactant molecules on different facets. b) Redox abilities of photogenerated charge carriers tuned by the surface electronic band structures of different facets. c) Accumulations of photogenerated electrons or holes on different facets. Reproduced with permission. ${ }^{[5]}$ Copyright 2017, Wiley.

The facets exposed on photocatalyst surface have been found to affect the photocatalytic activity through various mechanisms. For example, (1) surface atomic arrangements determine the adsorption and activation energy of reaction molecules, and thus affecting catalytic activity and selectivity (Figure 4a). ${ }^{[28,29]}$ (2) The surface electronic structures (i.e. surface chemical states), which vary with surface facets, provide the photogenerated charge carriers with different redox abilities for catalytic reactions (Figure 4b). ${ }^{[30]}$ (3) When the semiconductor is enclosed by multiple facets, the difference in electronic structures of surface facets result in the spatial charge separation which intrinsically separates the photogenerated electrons and holes on different facets for reduction and oxidation reaction, respectively (Figure 4c). ${ }^{[31-33]}$ These considerations have added exciting variables in tailoring morphology of nanoparticles (NPs) with preferentially exposed facets during the past decades. For instance, the spatial molecule adsorption/charge separation provides a driving force to selectively deposit the new components on the facets through reduction or oxidation reactions. ${ }^{[34]}$ As shown by Cheng's group, Au nanoparticles could be selectively photo-deposited on the lateral plane of $\mathrm{ZnO}$ nanosheets, in which the Au-based complex ions were preferentially attached to the negatively charged O-terminated (002) facet and then photo-reduced into Au nanoparticles. ${ }^{[35]}$ Also, Pt particles from $\mathrm{Pt}^{4+}$ photo-reduction were found mainly deposited on anatase $\mathrm{TiO}_{2}$ 
(101) facets, while $\mathrm{PbO}_{2}$ particles from $\mathrm{Pb}^{2+}$ photo-oxidation are mostly found on anatase $\mathrm{TiO}_{2}(001)$ facets. ${ }^{[36]}$

Unfortunately, facets with high reactivity are unstable and tend to diminish rapidly to minimize surface energy during the crystal growth. Accordingly, a structural directing specie (SDS) or surfactant is usually employed in the shape control of NPs, which renders them in metastable and high-energy forms. ${ }^{[37]}$ Those adsorbates which range from simple inorganic species, organic molecules to polymer can subtly modify the chemical state of surface features (especially metal cations) and provide a kinetic growth control for the formation of metastable shaped NPs. As the growth rate of a crystal facet depends on the surface energy (high-energy facets grow more quickly than low energy facets), different morphologies of NP could thus be prepared by using various structural directing molecules. ${ }^{[37]}$ In the facet engineering for decent photocatalysts, the simplest way is to increase the percentage of the exposed facets with higher photocatalytic performance. The high coverage of one facet on surface would facilitate the investigations on facet-dependent photocatalytic activity and related mechanisms. Accordingly, the understanding gained from the mechanism study can provide a guide for identifying the facet with high activity for increased photocatalytic performance. This rational approach has been widely used for the development of facet-engineered photocatalysis of transition metal oxide during the past decades.

\section{Different interpretations amongst researchers}

Usually, adsorbates (i.e. SDS or surfactant) employed in the shape control of NPs are removed before the photocatalytic testing to avoid its interference to the interested properties on this surface. However, in most cases, those so-called "clean surfaces" were not so clean as they claimed. Notice that residue adsorbates have been shown to remain on the facets and influence the adsorption of reactant molecules and, in particular, the chemical states of neighboring surface cations. ${ }^{[38]}$ Those adsorbates may also participate in photo-redox reaction and thus can interfere catalytic results. For example, they can act as sacrificial agents during water splitting, leading to their partial/total oxidation or be the source of carbon during $\mathrm{CO}_{2}$ photoreduction to methane or methanol. This forms a physical obstacle from reliably evaluating the facet-dependent photocatalytic performance. Also, from thermodynamic point of view, a new equilibrium between the uncapped high energy facet and its exposed environment (e.g. ambient) must have achieved to 
minimize the increased system energy. This stabilization usually accompanies with the generation of surface features such as oxygen vacancy $\left(\mathrm{V}_{\mathrm{o}}\right)$, hydroxyl group or cation with various chemical states, which play a decisive role in various applications (e.g. optoelectronic devices, heterogeneous (photo)catalysis). Given that each facet possesses distinctive intrinsic energy, it is understandable that both distribution and concentration of those surface features differ from facet to facet after reaching equilibrium with its exposed environment. Moreover, various processing steps used for shaped NPs from group to group further complicate the correlation of facet activity and thus always lead to different interpretations amongst researchers.

In our opinion, the blocking stone to deeper the understanding on the facet-dependent photocatalysis is the lack of proper surface characterization technique/method that can provide facet-based information on both qualitative and quantitative manner. For example, Huang's group prepared various morphologies (i.e. cube, rhombic dodecahedra and octahedra) of $\mathrm{Ag}_{2} \mathrm{O}^{[39]}$ and $\mathrm{Cu}_{2} \mathrm{O}^{[40]}$ that preferentially exposed (100), (110) and (111) facets, respectively. The cubic $\mathrm{Ag}_{2} \mathrm{O}$ showed the highest photoactivity toward the degradation of methyl orange molecules (MO), followed by octahedra and rhombic dodecahedra shapes. ${ }^{[39]}$ While, for $\mathrm{Cu}_{2} \mathrm{O}$ case, this order was reversed with no activity found for cubic $\mathrm{Cu}_{2} \mathrm{O} \cdot{ }^{[40]}$ However, very similar characterization results of both $\mathrm{Ag}_{2} \mathrm{O}$ and $\mathrm{Cu}_{2} \mathrm{O}$ cases were obtained by current spectroscopic approaches. It was assigned to the difficulty in revealing the observed strong facet effects by the current characterization techniques. This reflects the tip of iceberg in some semiconductor facet effects because the measured band energies do not necessary offer the best explanation or prediction of photocatalytic and electrical properties exhibited by the same materials but exposing different crystal faces. As the studies of titanium and zinc oxides ranked the top two among the first row of transition oxides during the past decades (Figure 5), we herein focus on their facet-dependent photocatalysis and corresponding characterization techniques adopted for activity correlation. 


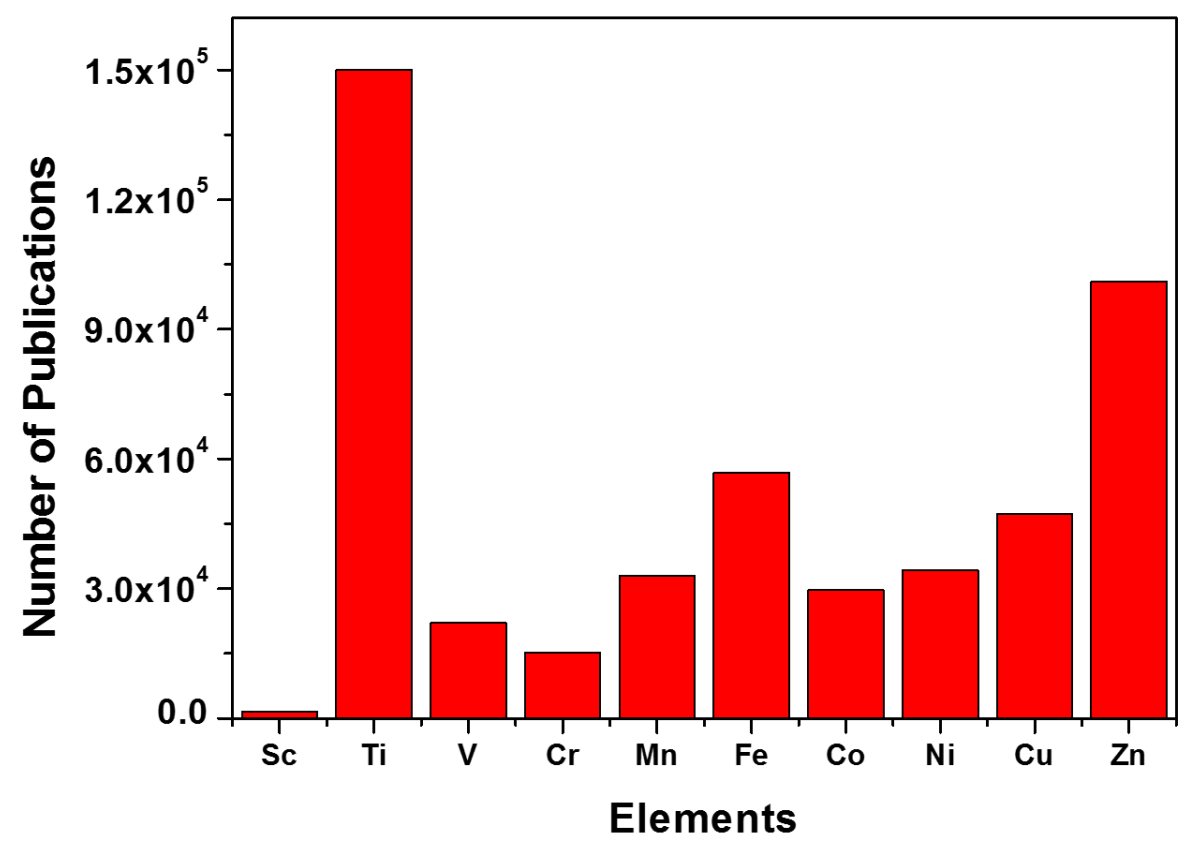

Figure 5. Comparison of total numbers of publications on different transition oxides since 2000. The data was collected based on Web of Science database using corresponding metal oxide as key word for search. 


\section{Facet-dependent photocatalysis of $\mathrm{ZnO}$ NPs and their characterization.}

a

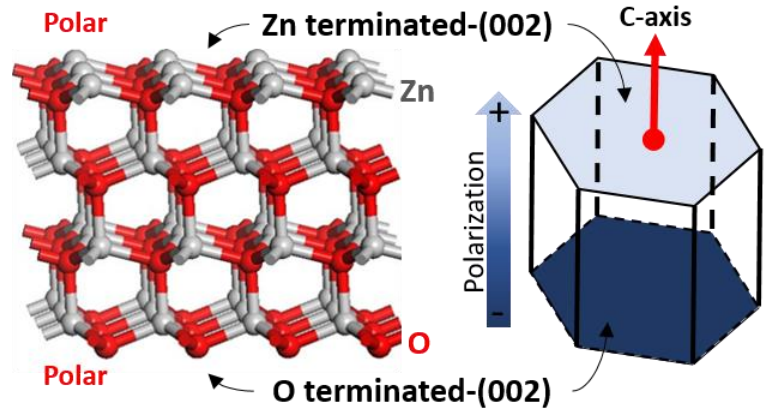

b

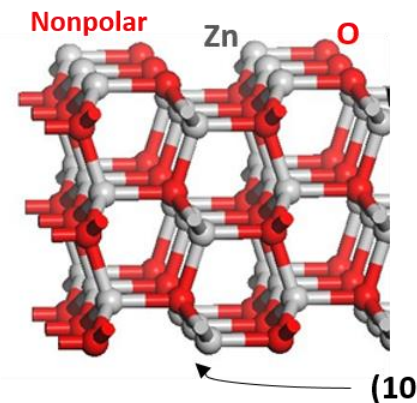

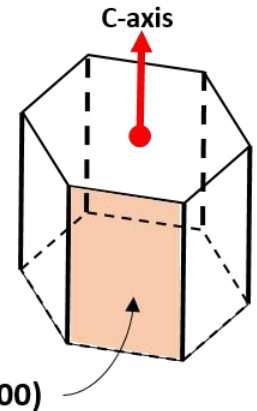

Figure 6. Schematic illustration of the hexagonal structure (wurtzite) of $\mathrm{ZnO}$. The three main crystallographic exposed planes and corresponding atomic arrangement are shown in (a) Zn-(002) and O-(002) facets with the intrinsic polarization field and (b) nonpolar (100) facet.

$\mathrm{ZnO}$ is a wide band gap semiconductor $(3.3 \mathrm{eV})$ with a high exciton binding energy of $60 \mathrm{meV}$ and piezoelectric properties. It is well known that $\mathrm{ZnO}$ is a polar crystal (wurtzite structure) composed of tetrahedrally coordinated $\mathrm{O}^{2-}$ and $\mathrm{Zn}^{2+}$ ions stacking along the $c$-axis and thus its surface consists of a positively charged Zn-terminated (002) plane (Zn-(002)), a negatively charged O-terminated (002) plane (O-(002)) (Figure 6a) and nonpolar planes (100) with $\mathrm{C}_{6 \mathrm{~V}}$ symmetry (Figure 6b). ${ }^{[41]}$ The specificity of polar surface comes from the combined effect of orientation and termination which is responsible for the existence of a macroscopic polarization along the surface normal and a surface electrostatic instability. Similar to the case of ferroelectric materials, a depolarization mechanism is required to stabilize these surfaces, which can be achieved in many ways: by a deep modification of the surface electronic structure (i.e. total or partial filling of surface states) or by strong changes in the surface stoichiometry (e.g. surface reconstruction, formation of surface defects or adsorption of foreign species). ${ }^{[41]}$ These processes may change the original surface configurations, in which the local environment of the surface atoms is very different from the bulk or non-polar facet, and new electronic surface states (e.g. defects) may appear in the bandgap of the metal oxide. Accordingly, tailoring ZnO NPs with preferential exposed polar (002) facets has been receiving increasing attention due to superior performance in solar cells and photocatalysis, ${ }^{[42-44]}$ methanol synthesis, ${ }^{[45,46]}$ optoelectronics ${ }^{[47]}$ and gas sensing, ${ }^{[48]}$ which were all attributed to increased concentration of oxygen vacancies $\left(\mathrm{V}_{\mathrm{o}}\right)$ on this face. Particularly, in the field of photocatalysis which is closely associated with the coordination environment and electronic states of the surface active atoms. 

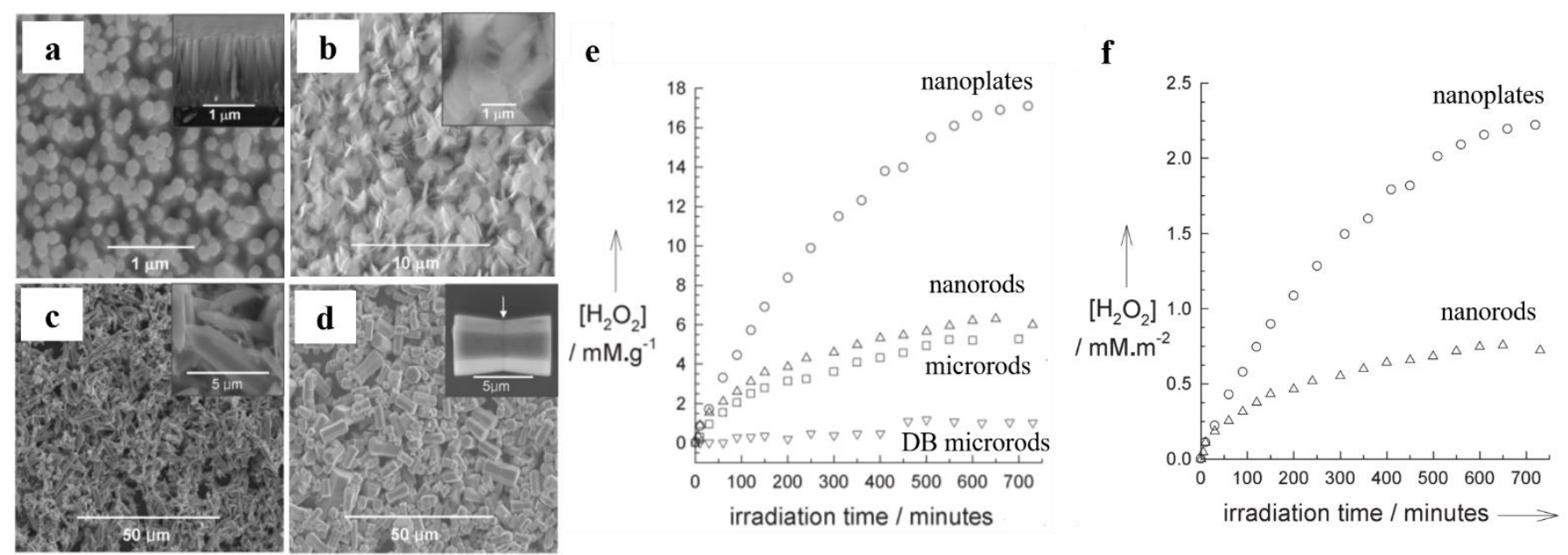

Figure 7. a-d) SEM images of $\mathrm{ZnO}$ (a) nanorods, (b) nanoplates, (c) microrods and (d) dumbbell microrods. (e) Weight-normalized and (f) surface-area-normalized time profiles of the evolution of $\mathrm{H}_{2} \mathrm{O}_{2}$ in UV-illuminated (wavelength $\lambda>300 \mathrm{~nm}$ ) suspensions. Reproduced with permission. ${ }^{[42]}$ Copyright 2006, Wiley.

The facet activity of polar $\mathrm{Zn}-(002)$ facet in the photocatalytic generation of $\mathrm{H}_{2} \mathrm{O}_{2}$ was firstly showed by Choy's group. ${ }^{[42]}$ This reaction was carried out with $10 \mathrm{mg}$ various $\mathrm{ZnO}$ morphologies using acetate as a hole scavenger under UV irradiation (300W Xe lamp) and oxygen bubbling. The evolution of $\mathrm{H}_{2} \mathrm{O}_{2}$ concentration was examined by an iodide titration through UV-vis absorption spectroscopy. They prepared various $\mathrm{ZnO}$ morphologies (Figure $7 \mathrm{a}-\mathrm{d}$ ) with calculated surface area of $\mathrm{Zn}$-(002) facet (based on SEM images $)$ in the order of nanoplates $\left(3.53 \mathrm{~m}^{2} / \mathrm{g}\right)$, nanorods $\left(0.12 \mathrm{~m}^{2} / \mathrm{g}\right)$, microrods $\left(0.03 \mathrm{~m}^{2} / \mathrm{g}\right)$ and dumbbell microrod $\left(0 \mathrm{~m}^{2} / \mathrm{g}\right)$. Notice that the dumbbell microrod masked $\mathrm{Zn}-(002)$ faces (indicated by white arrow in Figure $7 \mathrm{~d}$ inset) and exposed only O-(002) faces at both ends. The area of the exposed polar Zn-(002) facet was found proportional to the photocatalytic $\mathrm{H}_{2} \mathrm{O}_{2}$ generation normalized either by weight (Figure 7e) or by surface area (Figure 7f). However, using electron microscopy as a major characterization technique to establish the correlation of photocatalytic performance to facet activity is far from enough without controversy. As $\mathrm{H}_{2} \mathrm{O}_{2}$ is widely known to be unstable in ambient conditions, several blank experiments such as $\mathrm{H}_{2} \mathrm{O}_{2}$ decomposition with/without UV irradiation and in the presence or absence of $\mathrm{ZnO}$ should be carried out for a more accurate comparison. For example, $\mathrm{H}_{2} \mathrm{O}_{2}$ can self-decompose in the presence of $\mathrm{H}_{2} \mathrm{O}_{2}$ or metal ions and the decomposition rate of $\mathrm{H}_{2} \mathrm{O}_{2}$ on $\mathrm{ZnO}$ without irradiation may be facet-dependent. ${ }^{[49]}$ Also, it has been shown that the rate of $\mathrm{H}_{2} \mathrm{O}_{2}$ decomposition under UV irradiation can be up to $4.5 \mu$ molh $^{-1}$, ${ }^{50]}$ which is considerably high compared to Choy's result. ${ }^{[42]}$

For decades, a high $\mathrm{V}_{\mathrm{o} \text { surface }}$ concentration and its relation to exposed (002) facets have been regarded as the main criterion for high photocatalytic activity. It would be ideal to correlate the measured 
photocatalytic activity directly with the $\mathrm{V}_{\mathrm{o} \text { surface }}$ concentration in quantitative manner. However, the $\mathrm{V}_{\mathrm{o} \text { surface }}$ of $\mathrm{ZnO}$ is highly unstable when in contact with environment (i.e. $\mathrm{H}_{2} \mathrm{O}$ to favor the formation of $\mathrm{OH}$ surface or $\mathrm{O}_{2}$ for surface adsorption). This high affinity say for water molecule to $\mathrm{V}_{\mathrm{o}}$ surface on $\mathrm{ZnO} \mathrm{O}-(002)$ was indeed observed experimentally by Diebold et al. using STM and scanning tunneling spectroscopy (STS). ${ }^{[51]}$ Accordingly, the mechanism proposed by Jang et al. ${ }^{[42]}$ and McLaren et al. ${ }^{[44]}$ that the preferentially adsorbed $\mathrm{OH}^{-}$on positive charged polar surface might be the reason for enhanced photocatalytic activity. These ions can react with photo-generated holes $\left(\mathrm{h}^{+}\right)$to produce reactive species $\cdot \mathrm{OH}$ radicals, hence enhancing the photocatalytic activity. Nowadays, the photocatalytic mechanism of $\mathrm{ZnO}$ is still not clear mainly due to the lack of appropriate technique to provide quantitative $\mathrm{V}_{\mathrm{o} \text { surface }}$ and $\mathrm{OH}$ correlation in relevant to facet-dependent properties of $\mathrm{ZnO}$.

\subsection{Using PL as a main facet-characterization technique.}
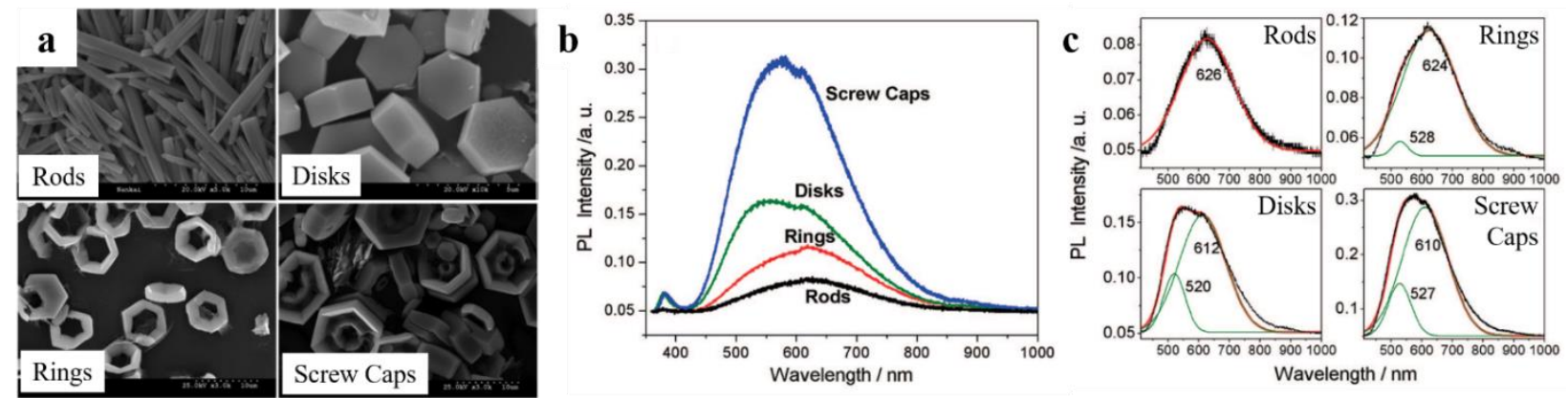

d

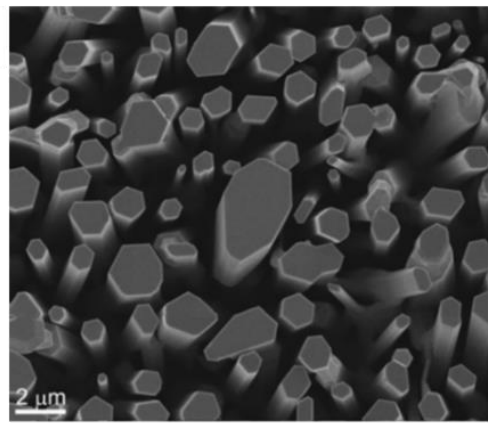

$\mathbf{e}$
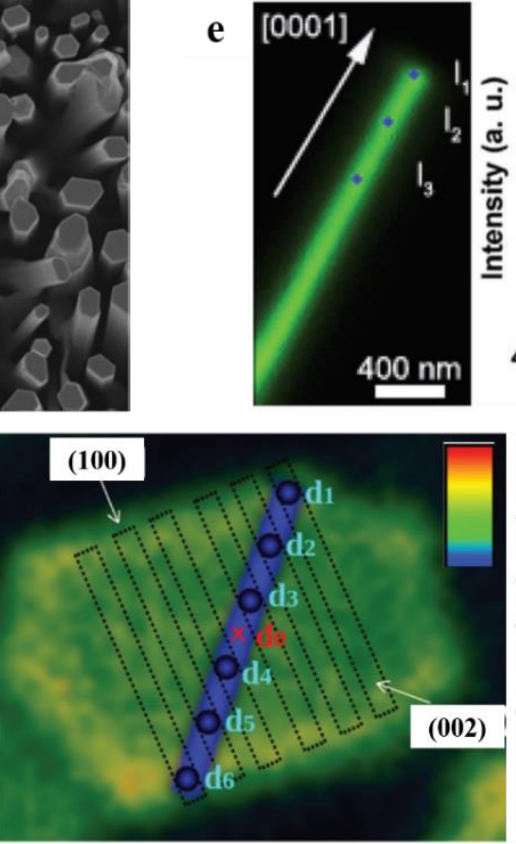
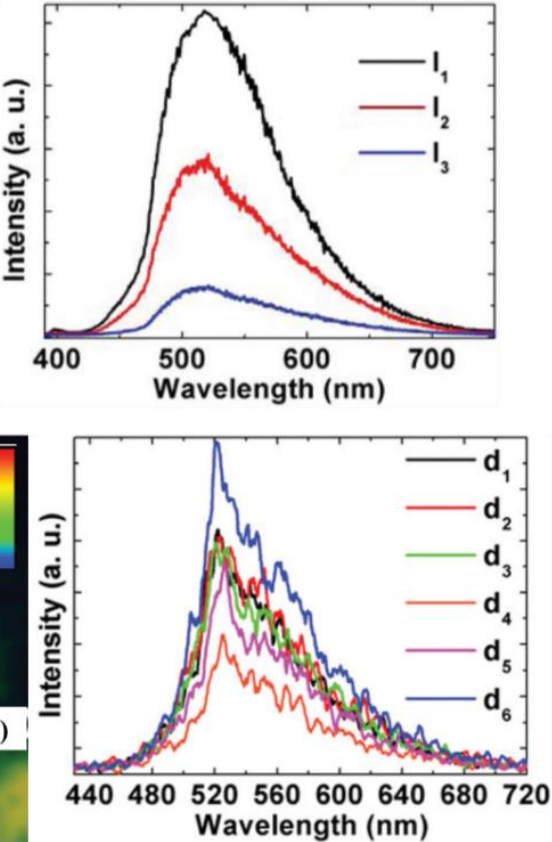

f

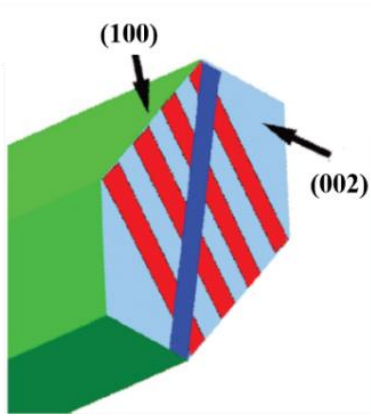

Figure 8. (a) SEM images of the synthesized $\mathrm{ZnO}$ with various morphologies: rods, disks, rings and screw caps. (b) PL spectra and (c) corresponding Guassian fit results of the 410-1000 nm emissions of the synthesized $\mathrm{ZnO}$ with various morphologies. Reproduced with permission. ${ }^{[43]}$ Copyright 2008, American Chemical Society. (d) Top SEM view of $\mathrm{ZnO}$ nanowire (NW) array. (e) Confocal PL intensity microscope 
image of a thin $\mathrm{ZnO} \mathrm{NW}$ lying on its side and corresponding PL spectra at three different spatial locations ( $\mathrm{I}_{1}$ to $\mathrm{I}_{3}$ ) along the [0001] direction. (f) Confocal intensity images of an individual $\mathrm{ZnO} \mathrm{NW}$ on the (002) surface and corresponding PL spectra of the green luminescence taken at six spatial locations $\left(\mathrm{d}_{1}\right.$ to $\left.\mathrm{d}_{6}\right)$ along the blue guide line. Reproduced with permission. ${ }^{[58]}$ Copyright 2013, American Institute of Physics.

To probe bulk defect sites, photoluminescence spectroscopy (PL) at short excitation wavelength has been commonly employed to characterize transition metal oxide semiconductors. These materials can capture the photons from the excitation source with energy equal to or greater than their band gap energy. The photons are then absorbed by the oxide semiconductor nanocrystal and cause the generation of photoexcited electrons and holes in the conduction and valence bands, respectively. The excited electronhole pairs, known as excitons, are then subject to recombination, in particular, on the defect centers to give trap-state luminescence. As bulk oxygen vacancy $\left(\mathrm{V}_{\mathrm{o} \text { bulk }}\right)$ is linked with the green-yellow luminescence of $\mathrm{ZnO}$, Li et al. first extended such a correlation to the surface oxygen vacancy $\left(\mathrm{V}_{\mathrm{o} \text { surface }}\right)$ concentration on polar $\mathrm{ZnO}$ facets monitored by using PL spectroscopy. ${ }^{[43]}$ They compared the facet catalytic activity of $\mathrm{ZnO}$ rods, disks, rings, and screw caps (Figure 8a) with their corresponding PL spectra (Figure 8b) and found a positive correlation between the PL intensity and the proportion of exposed (002) facet in the order of screw caps $>$ disks $>$ rings $>$ rods. Moreover, as the green emission $(\sim 520 \mathrm{~nm})$ has been reported a good correlation with the $\mathrm{V}_{\mathrm{o}}$ bulk and the yellow emission $(\sim 620 \mathrm{~nm})$ could be assigned to the interstitial oxygen, the PL broadband was thus further divided into two bands in the green and the yellow range (Figure 8c). The intensity of the green emission clearly varies with different $\mathrm{ZnO}$ morphologies, following the same order as the broadband intensity: screw caps $>$ disks $>$ rings $>$ rods. ${ }^{[43]}$ Also, it is widely accepted that the $\mathrm{V}_{\mathrm{o} \text { surface }}$ can act as potential wells to trap either one or two electrons, aiding electron-hole pair separation and thus increasing the photocatalytic activity. Since then, PL has become widely used as one of the major characterizaion techniques to evaluate the $\mathrm{V}_{\mathrm{o} \text { surface }}$ concentration of ZnO NPs in photocatalysis ${ }^{[43,52-55]}$ or optical/electronics. ${ }^{[56,57]}$

Recently, the preferential distribution of $\mathrm{V}_{\mathrm{o} \text { surface }}$ on the polar (002) facet of $\mathrm{ZnO}$ nanowire (NW) (Figure 8d) (cf. nonpolar (100) facet) was further demonstrated by Wong et al. using room-temperature confocal microscopy. ${ }^{[58]}$ Figure 8 e shows the confocal PL emission intensity of an individual ZnO NW lying on a side composed of the (100) facet surface and corresponding PL spectra at three different spatial locations $\left(\mathrm{I}_{1}\right.$ to $\left.\mathrm{I}_{3}\right)$ along the [0001] direction. The shape and location of the PL peaks remain unchanged and 
only the peak intensity increases $\left(I_{1}>I_{2}>I_{3}\right)$ with its location close to the tip of NW (i.e. where polar (002) facet is exposed). Also, Figure 8f shows the confocal PL spectra at six different spatial locations indicated by the blue line in the top cross-sectional (002) plane of the NWs. The intensity of the green luminescence peak was also found strongest at top edge (i.e. $d_{6}$ and $d_{1}$ ) where the (002) and (100) surfaces meet each other. ${ }^{[58]}$ Accordingly, their results qualitatively demonstrate the $V_{o}$ surface is populated on (002) facet (cf. (100) facet) especially at the interface between (002) and (100) facets.
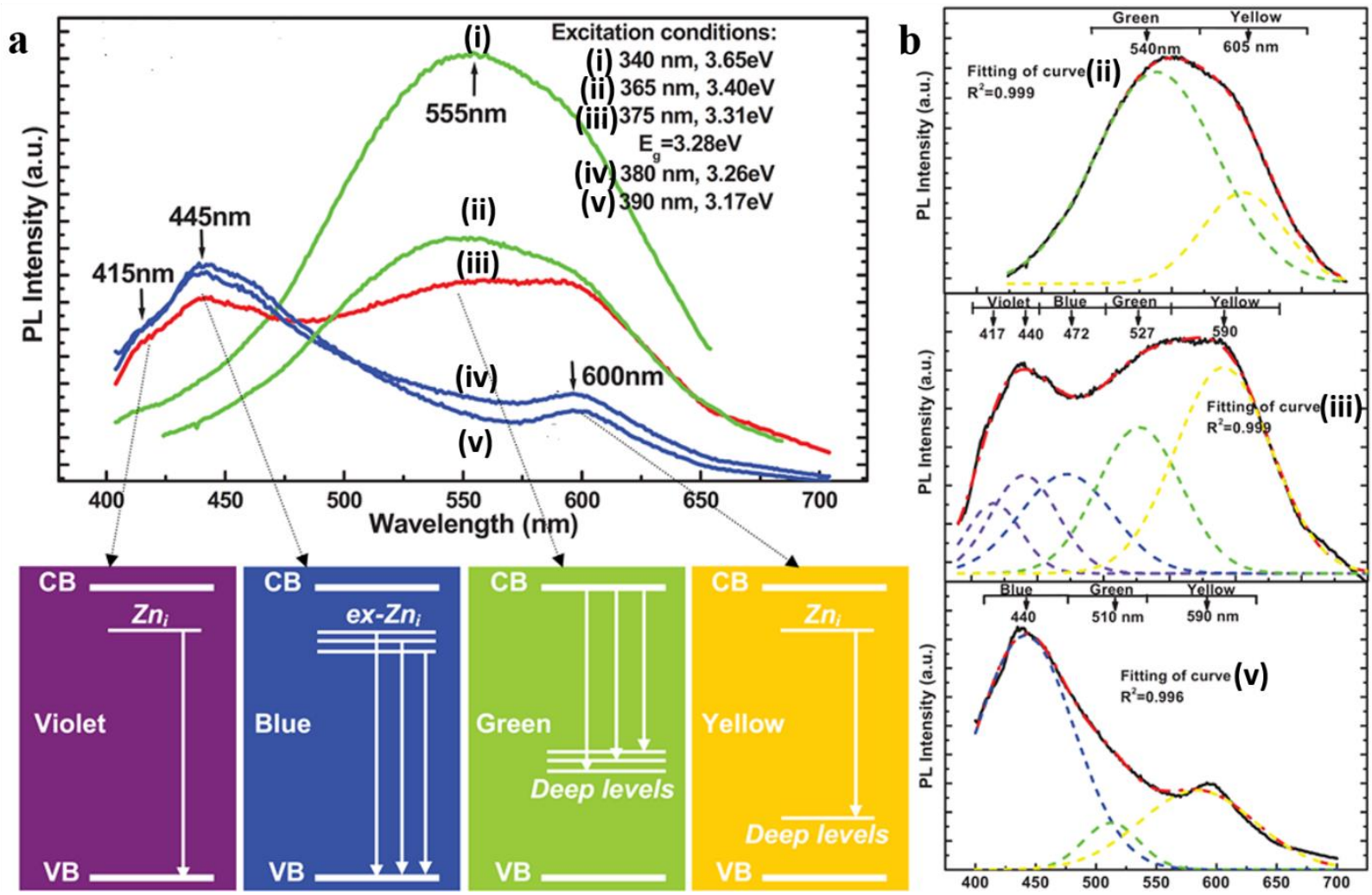

Figure 9. (a) $\mathrm{PL}$ spectra of $\mathrm{ZnO}$ nanoparticle $\left(\mathrm{E}_{\mathrm{g}}=3.28 \mathrm{eV}\right)$ with various excitation wavelength from (i) $3.65 \mathrm{eV}$ to $(\mathrm{v}) 3.17 \mathrm{eV}$. The proposed mechanisms are denoted for violet, blue, green, and yellow emissions, respectively. (b) Spectra deconvolution of (ii), (iii) and (v) based on the proposed mechanism. Reproduced with permission. ${ }^{[57]}$ Copyright 2010, Wiley.

However, as stated above, PL is not a surface-specific technique and thus fails to deliver precise $\mathrm{V}_{\mathrm{o}}$ surface with interferences from bulk defects (e.g. Zn interstitial). Moreover, as shown by Zeng et al., the PL pattern was found highly dependent on excitation wavelength used no matter the excitation energy is larger (i.e. (i) to (iii)) or smaller (i.e. (iv) and (v)) than the bandgap (Figure 9a). Generally, the broad peak with sub-peaks at a longer wavelength than the corresponding band gap is achieved because of the various emissions from the defect levels between the bands. ${ }^{[57]}$ According to Zeng et al., the violet ( 415 nm) and blue $(\sim 445 \mathrm{~nm})$ emissions in Figure 9a are attributed to the transitions from $\mathrm{Zn}$ interstitial-related defects $\left(\mathrm{Zn}_{\mathrm{i}}\right)$ and extended $\mathrm{Zn}_{\mathrm{i}}\left(\mathrm{ex}-\mathrm{Zn}_{\mathrm{i}}\right)$ located slightly below the conduction band edge to the valance band, 
respectively. ${ }^{[57]}$ Furthermore, the green $(\sim 530 \mathrm{~nm})$ and yellow $(\sim 600 \mathrm{~nm})$ regions represent the transitions from the conduction band and $\mathrm{Zn}_{\mathrm{i}}$ to deep levels ( $\mathrm{V}_{\mathrm{o} \text { bulk }}$ of $\sim 1 \mathrm{eV}$ above the valance band), respectively (Figure 9a). The Gaussian fitting in Figure 9b clearly shows that even though curve (ii) and curve (iii) were obtained with the excitation energy larger than the bandgap, both the intensity and position of green emission varies with excitation energy. With the excitation energy decreasing, the green emission weakens but a new emission in violet-blue region emerges and increases. When the excitation energy is near the bandgap (i.e. curve (iii)), a very broad emission can be obtained in which the violet-blue and green emissions have comparable intensity. As the excitation energy decreases below the bandgap (i.e. curve (v)), most of the green emission is quenched except for a weak shoulder at $600 \mathrm{~nm}$. As a result, the spectra are then dominated by the violet-blue emission.

Due to this excitation-dependent property and the interferences from bulk defects especially $\mathrm{Zn}_{\mathrm{i}}$, a single PL determination is therefore not enough to provide concrete reliable correlation between $\mathrm{V}_{\mathrm{o}}$ surface and photocatalytic performance. ${ }^{[59]}$ Often, the comparison of such green emission from $\mathrm{V}_{\mathrm{o} \text { surface }}$ is also limited to individual studies, as the processing and post-treatment techniques used in the preparation of ZnO NPs vary from group to group. ${ }^{[60]}$ This results in many differences between nanoparticles, in addition to the exposed facet, such as the concentration of bulk defects and the particle size (may affect scattering). Cross-literature comparison of catalysts performance is thus seriously hindered as there is currently no universal technique which can provide quantitative analysis of the concentration/distribution of $\mathrm{V}_{\mathrm{o} \text { surface }}$ and the percentage of the desired facet on a given catalyst.

\subsection{Using EPR as a main facet-characterization technique.}
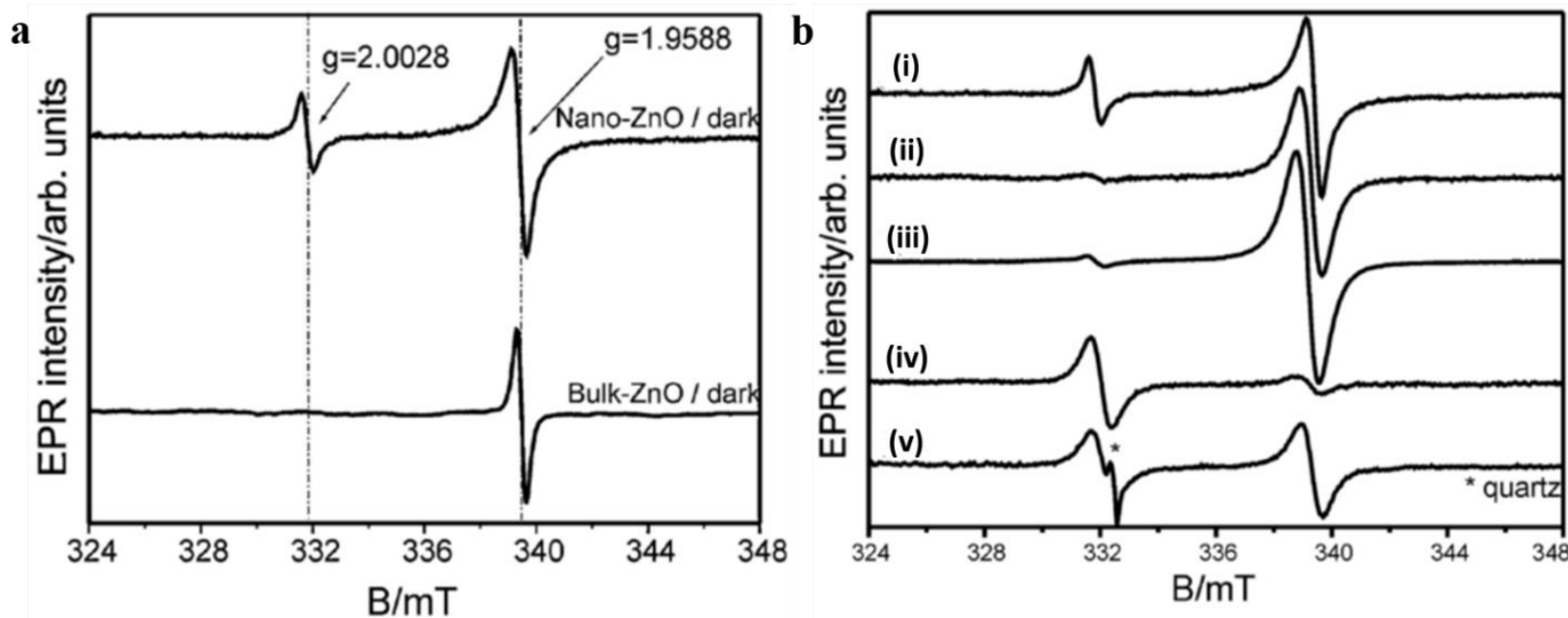
Figure 10. (a) X-Band EPR spectra of bulk- and nano-sized $\mathrm{ZnO}$ particles. (b) X-Band EPR spectra of nano-sized $\mathrm{ZnO}$ as treated in the following sequence: (i) initial, (ii) after exposure to air (adsorption of water molecules), (iii) after irradiation with UV light, (iv) after 5 min heating at $150^{\circ} \mathrm{C}$, (v) after UV irradiation. The signal designated by an asterisk (*) is owing to a signal from the EPR quartz tube. Reproduced with permission. ${ }^{[64]}$ Copyright 2009, Royal Society of Chemistry.

EPR spectroscopy is another technique that is widely employed to evaluate the $\mathrm{V}_{\text {o surface }}$ of $\mathrm{ZnO}$ NPs through the monitor of the transition of unpaired electron spins. ${ }^{[52,53,57,61-65]}$ As shown in Figure 10a, both bulk and nano-sized $\mathrm{ZnO}$ reveal an intense signals at approximately $\mathrm{g}=1.96$. Its origin is attributed to the unpaired electrons trapped by shallow donors or impurities. ${ }^{[22,64,65]}$ For nano-sized $\mathrm{ZnO}$, an additional resonance line at approximately $\mathrm{g}=2.00$ is assigned to the unpaired electrons trapped in $\mathrm{V}_{\mathrm{o} \text { surface }}$ by adsorbed dioxygen $\left(\mathrm{O}_{2}{ }^{-}\right)$or other low-coordinate surface defect sites. ${ }^{[22,64,65]}$ In general, these results are referenced to the bulk-surface (core-shell) model of $\mathrm{ZnO}$ that the concentration ratio between core and shell (surface-to-volume ratio) is defined for a given particle size. Accordingly, these two observed unpaired electron spins of the nano-sized $\mathrm{ZnO}$ can be realized that the signal at $\mathrm{g}=1.96$ is due to the bulk (core) contribution and the one at $\mathrm{g}=2.00$ originates from the surface (shell). ${ }^{[64]}$ As a quantitative value [spin counts* $\mathrm{g}^{-1}$ of sample] can be obtained by the integration of an EPR signal (i.e. the concentration of trapped unpaired electron), the cross-comparison of $\mathrm{V}_{\mathrm{o} \text { surface }}$ concentration between literature data is thus feasible. On the other hand, the surface EPR signal at $\mathrm{g}=2.00$ was also found strongly dependent upon humidity. As shown in Figure 10b(ii), this signal almost disappears while the signal intensity of the bulk signal (i.e. $\mathrm{g}=1.96$ ) remains unaffected. Comparing to the tiny increase of $\mathrm{g}=2.00$ by UV illumination (Figure $10 \mathrm{~b}(\mathrm{iii})$ ), a considerable increase of $\mathrm{g}=2.00$ was observed after later thermal treatment at the expense of $\mathrm{g}=1.96$ due to desorption of water molecule (Figure $10 \mathrm{~b}$ (iv)). The intensity of $\mathrm{g}=1.96$ can also be increased after a second illumination with UV light of the heat-treated sample (Figure 10b(v)). ${ }^{[64]}$ 
a

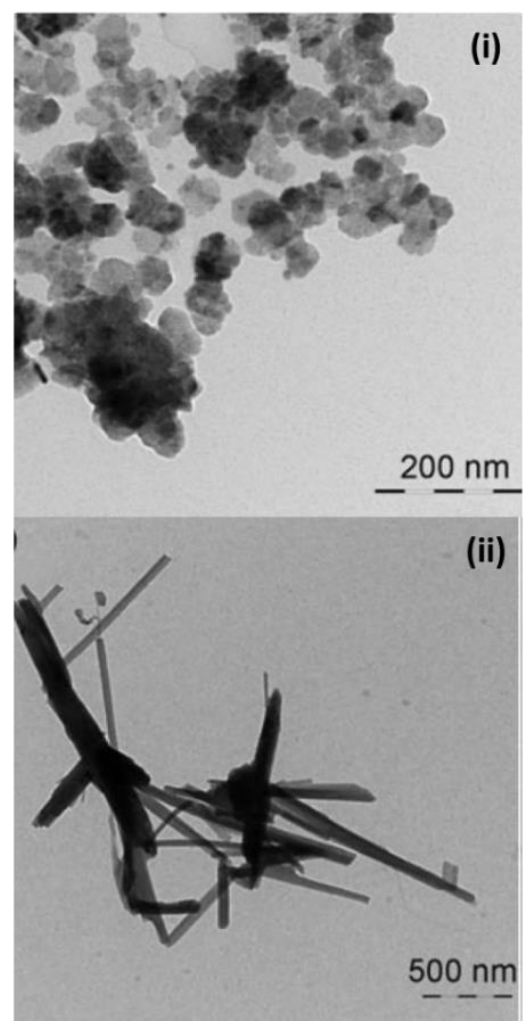

b

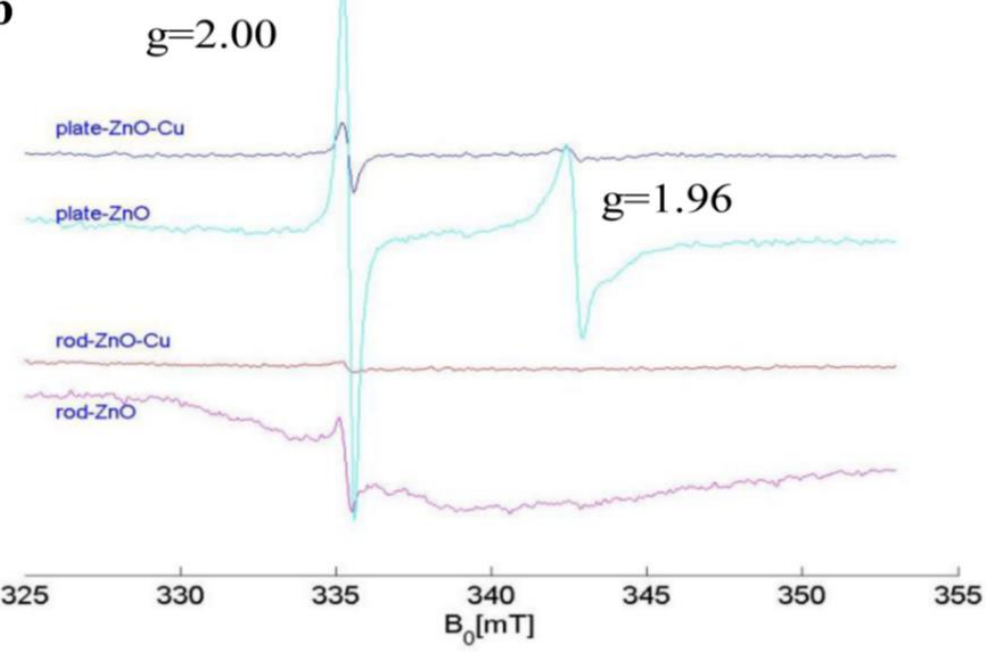

\begin{tabular}{lll}
\hline Sample & $\begin{array}{l}\text { Spin count }\left[\mathrm{mg}^{-1} \mathrm{ZnO}\right], \\
\mathrm{g}=2.00\end{array}$ & $\begin{array}{l}\text { Spin count }\left[\mathrm{mg}^{-1} \mathrm{ZnO}\right] \\
\mathrm{g}=1.96\end{array}$ \\
\hline Rod $\mathrm{ZnO}$ & $2.05 \times 10^{13}$ & 0 \\
$\mathrm{Cu} /$ rod $\mathrm{ZnO}$ & $2.71 \times 10^{13}$ & 0 \\
Plate $\mathrm{ZnO}$ & $6.98 \times 10^{13}$ & $1.51 \times 10^{14}$ \\
$\mathrm{Cu} /$ plate $\mathrm{ZnO}$ & $6.14 \times 10^{13}$ & $2.53 \times 10^{13}$ \\
\hline
\end{tabular}

Figure 11. (a) TEM images of $\mathrm{ZnO}$ (i) nanoplate and (ii) nanorods. (b) EPR spectra of $\mathrm{ZnO}$ nanoparticles (plate, rod and their mixtures with copper) and corresponding quantitative table of peaks at $\mathrm{g}=2.00$ and g=1.96. Reproduced with permission. ${ }^{[22]}$ Copyright 2011, Wiley.

Similar to the PL observation, a correlation between the intensity of EPR g value at 2 (i.e. $V_{\text {o surface }}$ concentration) and polar $\mathrm{ZnO}$ facets was firstly illustrated by Liao et al. ${ }^{[22]}$ They compared the EPR signals of plate-shape (Figure 11a(i)) and rod-shape (Figure 11a(ii)) ZnO NPs with preferential exposed polar (002) and nonpolar (100) facets and found a positive correlation of exposed (002) facet and the intensity of $g=2.00$ in the order of nanoplate $\left(6.98 * 10^{13} \mathrm{spins} / \mathrm{mg}\right)>\operatorname{nanorod}\left(2.05^{*} 10^{13} \mathrm{spins} / \mathrm{mg}\right)$ (Figure $\left.11 \mathrm{~b}\right)$. They also presented the significance of polar $\mathrm{ZnO}(002)$ facet on its electronic interaction with copper in the synthesis of methanol from carbon dioxide hydrogenation. The electron transfer from the conduction band of $\mathrm{ZnO}$ nanoplate to copper is evident from the decrease of both signal at $g=2.00$ and $g=1.96$ after the addition of copper (Figure 11b). ${ }^{[2]}$ As illustrated above, this EPR technique seems promising and much precise than previous PL technique since no interference from bulk species and a quantitative result of $\mathrm{V}_{\mathrm{o}}$ surface concentration makes cross-literature comparison feasible.

However, according to Walters et al.'s experiment, the signal at $\mathrm{g}=2.00$ cannot represent the true $\mathrm{V}_{\mathrm{o}}$ surface concentration as only $\mathrm{O}_{2}$ adsorbed $\mathrm{V}_{\mathrm{o} \text { surface }}$ with one $\left(\left(\mathrm{V}_{\mathrm{o} \text { surface }}{ }^{+}\right)^{-}\right)$or two $\left(\left(\mathrm{V}_{\mathrm{o} \text { surface }}\right)^{2-}\right)$ free electrons contributes to this signal and no overall signal for $\mathrm{V}_{\mathrm{o} \text { surface }}$ with no electron left in its ionic form $\left(\mathrm{V}_{\mathrm{o} \text { surface }}\right.$ $\left.{ }^{2+}\right) .{ }^{[61-63]}$ Also, if we consider the equation $\mathrm{O}_{2}+\mathrm{V}_{\mathrm{o} \text { surface }}+\mathrm{e}^{-} \leftrightarrow \mathrm{O}_{2}^{-}\left(\mathrm{V}_{\mathrm{o} \text { surface }}\right)$, not all $\left(\mathrm{V}_{\mathrm{o} \text { surface }}{ }^{-}\right)^{-}$and $\left(\mathrm{V}_{\mathrm{o}}\right.$ 
surface $)^{2-}$ sites could take up $\mathrm{O}_{2}$ due to the equilibrium involved. ${ }^{[61-63]}$ Accordingly, EPR is thus a semi-quantitative technique for $\mathrm{V}_{\mathrm{o} \text { surface }}$ and no knowledge of facets and other surface features are actually provided. As PL and EPR are not a truly facet-specific techniques, studies bridging the active facet and catalytic result may sometimes lead to disagreements as no information available on the distribution of surface features (e.g. $\mathrm{V}_{\mathrm{o}}$ here) among facets. ${ }^{[59]}$ A universal technique that can provide cross-literature comparison on the distribution/concentration of surface features among various facets is therefore highly desirable. As such, a comprehensive understanding of these acidic properties is crucial for the design, modification and applications of solid acid catalysts. 


\subsection{Using probe-assisted NMR as a potential facet-characterization technique.}
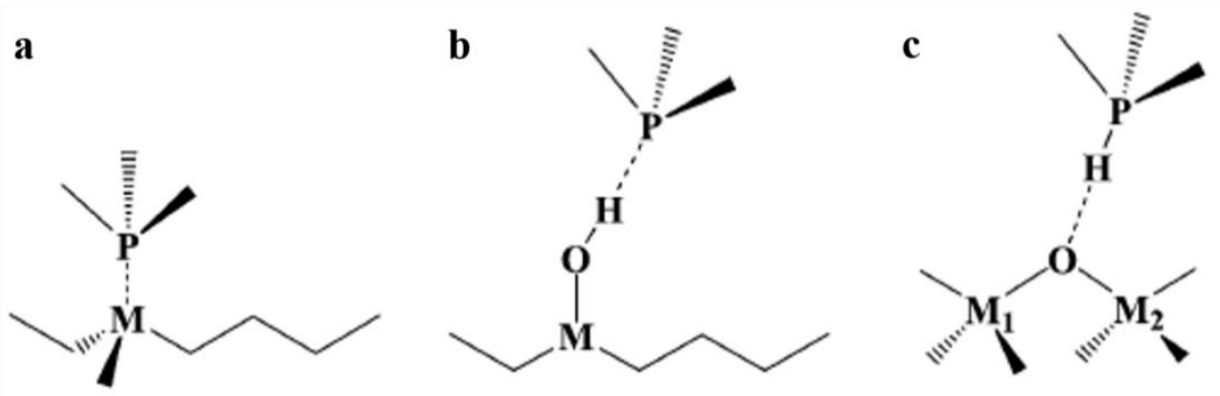

Figure 12. TMP molecule interacts with (a) metal cation (Lewis acid, LA), (b) isolated hydroxyl proton (physical interaction) and (c) bridging hydroxyl proton (Brønsted acid, BA). Reproduced with permission. ${ }^{\text {[44] }}$ Copyright 2010, Wiley.

Since metal ion sites exposed with the introduction of surface by $\mathrm{V}_{\mathrm{o} \text { surface }}$ can function as Lewis acid (LA) sites, conventional methods using basic probe molecules such as temperature-programmed desorption (TPD) of ammonia ${ }^{[66,67]}$ and Fourier-transform infrared spectroscopy $(F T I R)^{[67]}$ of pyridine have been widely used for characterization of catalyst's acidic features (e.g. acid type, strength and concentration). However, the probe molecules used in these cases are normally too basic to distinguish their differences between $\mathrm{V}_{\mathrm{o} \text { surface }}$ and other Lewis acid sites by their strength and distribution, leading to simple profiles of an averaged signal of LA sites. Taken pyridine-FTIR as an example, even though the type of Brönsted acid (BA) and LA site can be readily differentiated by the signal at 1540 and $1455 \mathrm{~cm}^{-1}$, no further information on the strength and distribution of BA/LA sites can be made available. Alternatively, probe-assisted nuclear magnetic resonance (NMR) has recently been shown its potential in differentiating various micro-environments by using corresponding chemical shift values. Among NMR-active basic probes (e.g. ${ }^{13}$ carbon monooxide for ${ }^{13} \mathrm{C}$, pyridine for ${ }^{15} \mathrm{~N}$, trimethylphosphine (TMP) for ${ }^{31} \mathrm{P} . .$. etc.), ${ }^{31} \mathrm{P}$ nucleus with $100 \%$ natural abundance and a chemical shift $\left(\delta^{31} \mathrm{P}\right)$ range over $430 \mathrm{ppm}$ has been shown a sensitive and reliable technique capable of providing qualitative information (the type (BA or LA) and strength of acid sites) and quantitative information (concentration of each site) in various solid acid catalysts such as microporous zeolites, mesoporous molecular sieves, supported/sulfated metal oxide catalyst. ${ }^{[68]}$ Figure 12 shows three scenarios of interactions between TMP and metal oxide. The $\delta^{31} \mathrm{P}$ of adsorbed TMP spans over a wide range (-20 -58 ppm) when interacting with LA centers (metal cations) with various acid strengths (Figure 12a), whereas a $\mathrm{TMPH}^{+}$ionic complex formed when a TMP molecule adsorbs onto a bridging hydroxyl proton (Figure 12c) tends to give rise to a ${ }^{31} \mathrm{P}$ resonance in a much narrower range of -2 to $-5 \mathrm{ppm}$. The relatively weak (physical) interaction between TMP and isolated hydroxyl proton gives a signal at 
higher field ( -61 ppm, Figure 12b). Therefore, Lewis acid (electron acceptor), Brønsted acid (proton donor) and physical-adsorbed sites on metal oxide surface can be readily distinguished using TMP by high resolution ${ }^{31} \mathrm{P}$ magic angle spinning (MAS) NMR. ${ }^{[68]}$ Even though this method can provide the information of BA and LA on metal oxides, the knowledge of the distribution of those sites on various facets, which is the key point to understand the shape-dependent activity, is still deficient.
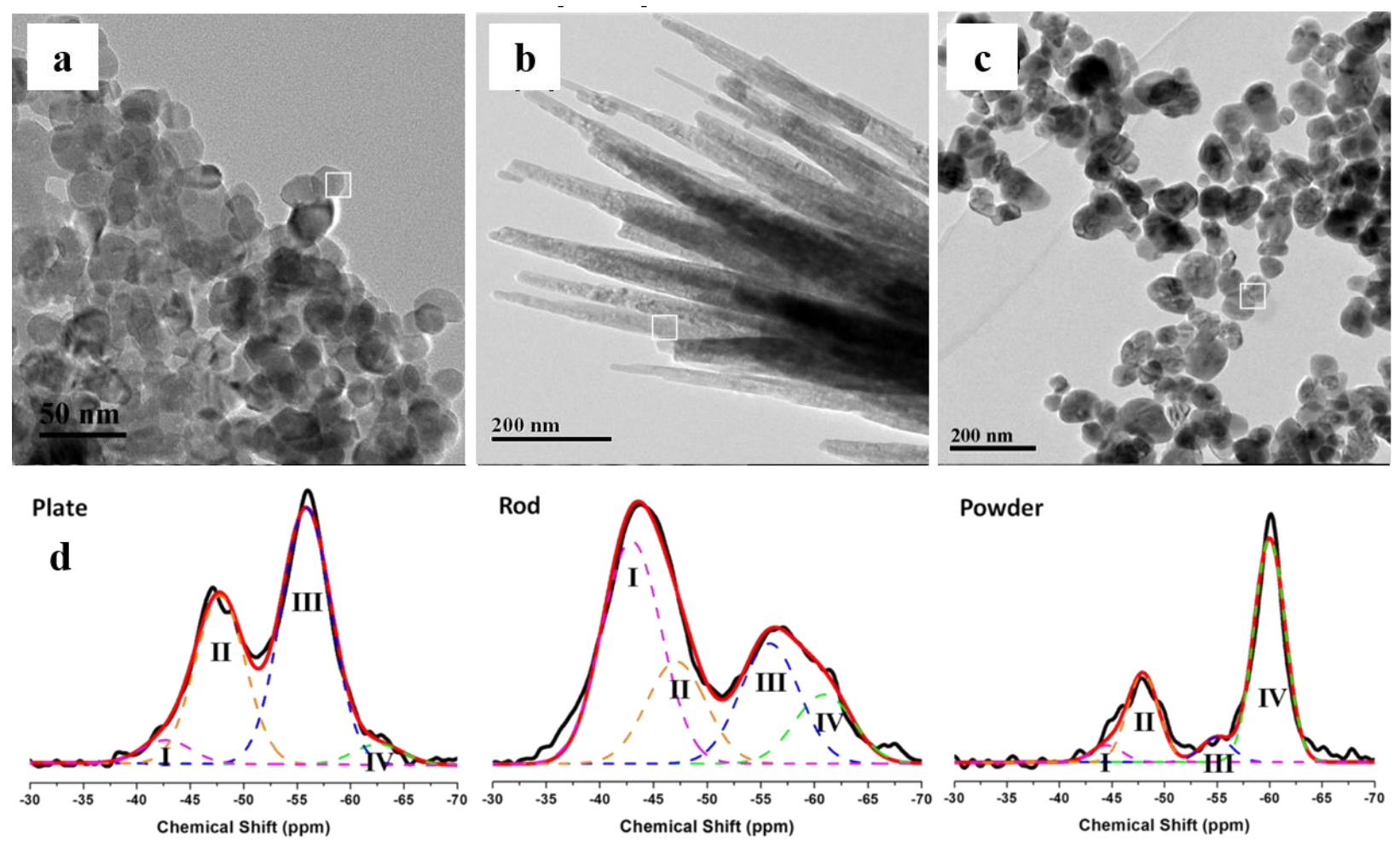

\begin{tabular}{|c|c|c|c|c|c|c|c|c|c|c|c|c|c|}
\hline \multirow{2}{*}{ ZnO } & \multicolumn{3}{|c|}{ Site I (-43 ppm) } & \multicolumn{3}{c|}{ Site II (-48 ppm) } & \multicolumn{3}{c|}{ Site III (-55 ppm) } & \multicolumn{3}{c|}{ Site IV (-61 ppm) } & \multicolumn{1}{c|}{ total } \\
\cline { 2 - 15 } & ppm & Area(\%) & TMP* & ppm & Area(\%) & TMP* & ppm & Area(\%) & TMP* & ppm & Area(\%) & TMP* & $\begin{array}{c}\text { Toverage of } \\
\text { OH }\end{array}$ \\
\hline Plate & -42.8 & 5.2 & 1.83 & -47.8 & 38.2 & 13.45 & -55.6 & 52.3 & 18.41 & -61.9 & 4.3 & 1.51 & 19.92 \\
\hline Rod & -42.9 & 43.8 & 16.01 & -47.1 & 19.9 & 7.27 & -55.5 & 23.2 & 8.48 & -60.9 & 13.1 & 4.79 & 13.27 \\
\hline Powder & -44.1 & 4.6 & 1.62 & -47.9 & 24.2 & 8.53 & -54.9 & 6.8 & 2.4 & -60.1 & 64.4 & 22.69 & 25.09 \\
\hline
\end{tabular}

$\mathbf{e}$

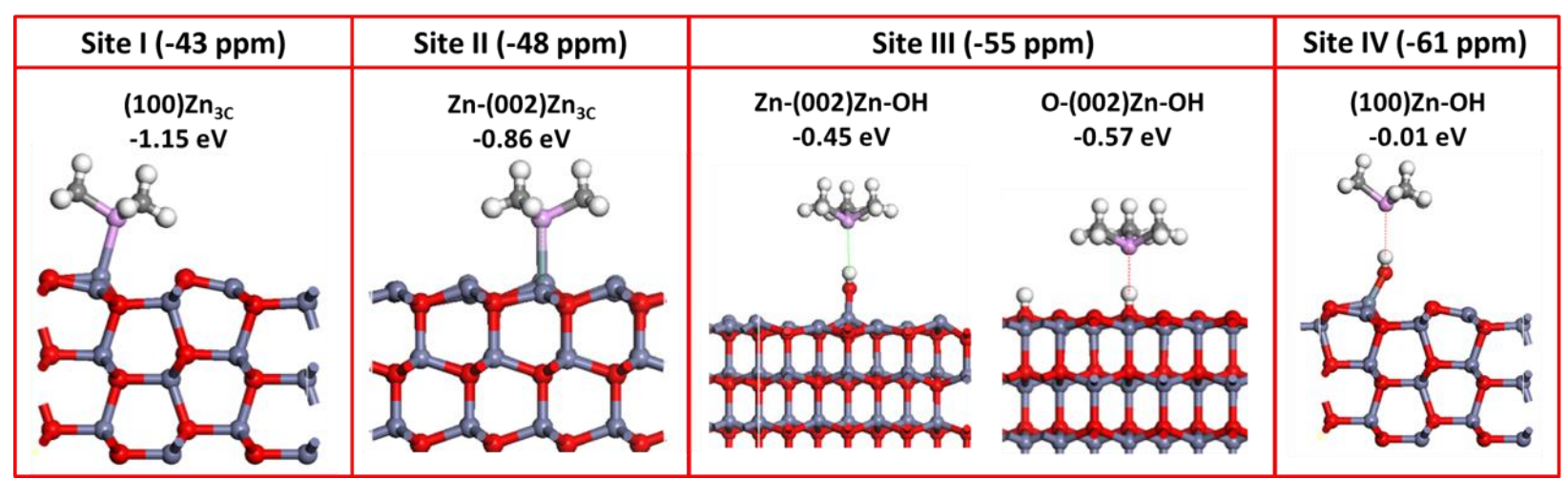

Figure 13. TEM images of $\mathrm{ZnO}$ (a) plate, (b) rod and (c) powder. (d) ${ }^{31} \mathrm{P}$ MAS NMR spectra of TMP adsorbed $\mathrm{ZnO}$ plate, rod, powder and corresponding spectral deconvolution. *Adsorbed TMP molecules in $\mu \mathrm{mol} / \mathrm{g}$. (e) Schematic illustrations of molecular interaction and calculated adsorption energy between TMP and various surface features: (100) $\mathrm{Zn}_{3 \mathrm{C}}, \mathrm{Zn}-(002) \mathrm{Zn}_{3 \mathrm{C}}, \mathrm{Zn} / \mathrm{O}-(002) \mathrm{Zn}-\mathrm{OH}$ and (100)Zn-OH. (100) $\mathrm{Zn}_{3 \mathrm{C}}$ : $\mathrm{Zn}_{3 \mathrm{C}}$ on (100) facet; (100)Zn-OH: hydroxyl group on (100) facet; $\mathrm{Zn}-(002) \mathrm{Zn}_{3 \mathrm{C}}: \mathrm{Zn}_{3 \mathrm{C}}$ on $\mathrm{Zn}$-terminated (002) facet; Zn-(002)Zn-OH and O-(002)Zn-OH: hydroxyl group on Zn-/O-terminated (002) facet, 
respectively. Reproduced with permission. ${ }^{[60]}$ Copyright 2016, American Chemical Society.

Given that each facet possesses its distinctive intrinsic energy, it is understandable that both concentration and chemical state of surface features differ from facet to facet. Recently, Tsang's group reported TMP-assisted surface mapping of $\mathrm{ZnO}$ nanoparticles and showed that it can provide comprehensive information (both qualitative and quantitative) on the surface features (e.g. BA, LA, isolated OH) of various facets. ${ }^{[60]}$ Single-crystalline plate- (Figure 13a) and rod- (Figure 13b) shaped ZnO nanoparticles with preferential exposed (002) and (100) facets, respectively and polycrystalline $\mathrm{ZnO}$ powder (Figure 13c), were prepared to study the correlation between $\delta^{31} \mathrm{P}$ and the adsorption configurations of TMP on various $\mathrm{ZnO}$ facets. Since no signal of $\mathrm{TMPH}^{+}(-2 \sim-5 \mathrm{ppm})$ is detected in all the three $\mathrm{ZnO}$ samples, the peaks at -43 , $-48,-55$ and $-61 \mathrm{ppm}$ can be initially assigned as the interactions between TMP and surface cations (Figure 13d). The spectrum of each $\mathrm{ZnO}$ sample, together with density function theory (DFT) calculation (Figure 13e), was deconvoluted into four components, namely Site I ( $-43 \mathrm{ppm})$, Site II ( $-48 \mathrm{ppm})$, Site III ( $-55 \mathrm{ppm})$ and Site IV (-61 ppm) and the concentration of each site can be quantified according to their corresponding peak area (Figure 13d). As nonpolar (100) and polar (002) facets dominate in the rod and plate sample, the experimentally observed resonance at around -43 (Site I) and $-48 \mathrm{ppm}$ (Site II) can be attributed to the interaction between TMP and $\mathrm{Zn}_{3 \mathrm{C}}$ on (100) facet $\left((100) \mathrm{Zn}_{3 \mathrm{C}}\right)$ and on $\mathrm{Zn}$-terminated (002) facet $\left(\mathrm{Zn}-(002) \mathrm{Zn}_{3 \mathrm{C}}\right)$, respectively. ${ }^{[60]}$ According to both experimental and theoretical data, the interaction between hydroxyl protons and TMP were also found highly dependent on the surface polarity of the $\mathrm{ZnO}$ facet. For example, the adsorption of TMP on polar $\mathrm{Zn}-(002) \mathrm{Zn}-\mathrm{OH}$ and $\mathrm{O}-(002) \mathrm{Zn}-\mathrm{OH}$ is much stronger with $\delta^{31} \mathrm{P}$ at $-55 \mathrm{ppm}$ while its interaction with nonpolar (100) $\mathrm{Zn}-\mathrm{OH}$ gives is weaker with $\delta^{31} \mathrm{P}$ at $-61 \mathrm{ppm}$ (Figure 13e). This preferential adsorption of molecules on a specific facet is attributed to the difference in surface energy/atomic arrangement between facets and, most importantly, can be readily distinguished by NMR chemical shift. ${ }^{[60]}$ As the surface coverage of proton is correlated with $\mathrm{V}_{\mathrm{o} \text { surface }}$ by the recombination of $\mathrm{H}$ atoms with $\mathrm{OH}$ species (i.e., thermal desorption of water), the temperature-dependent $\mathrm{V}_{\mathrm{o}}$ surface concentration on polar (002) can be calculated by this technique. ${ }^{[60]}$ 

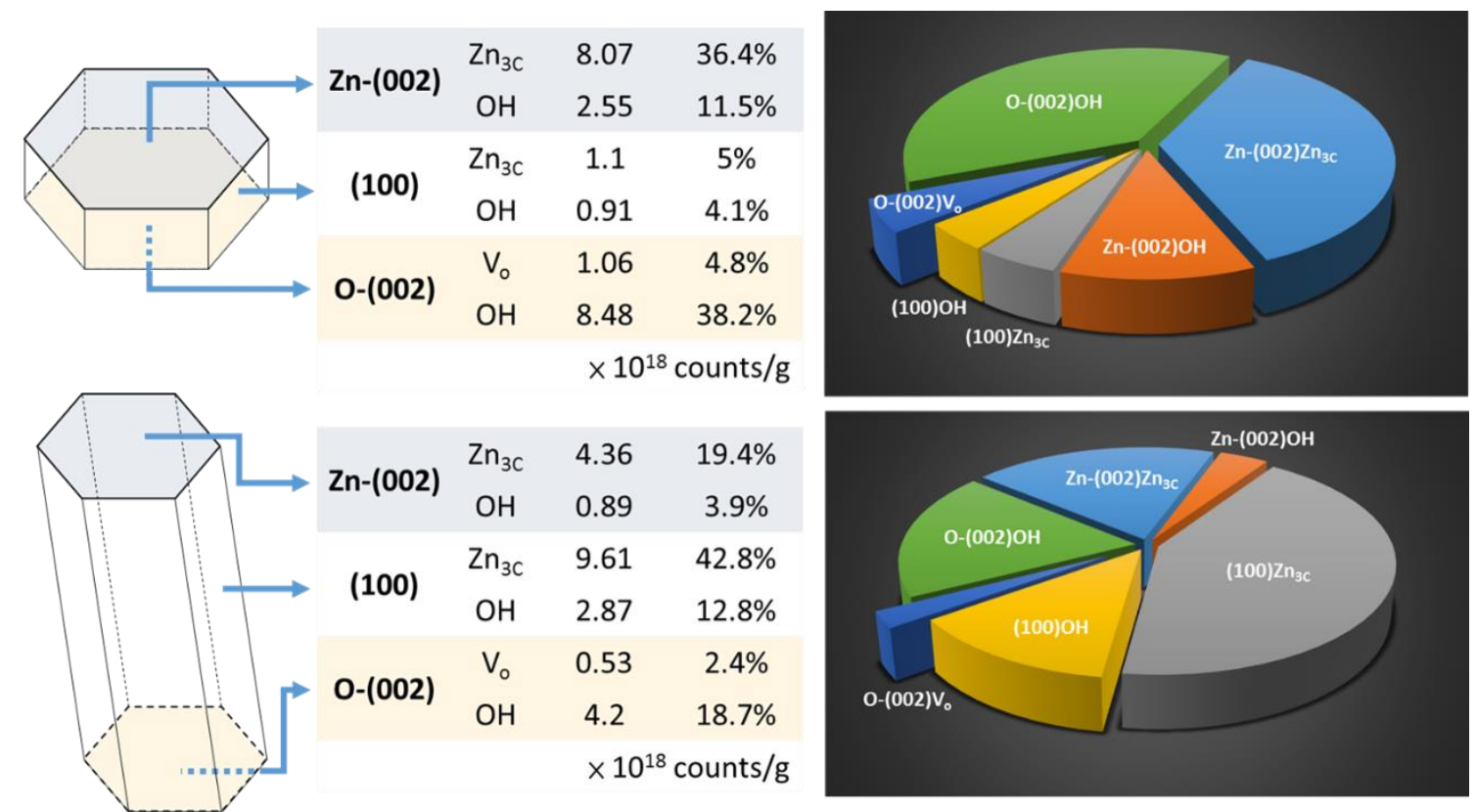

Figure 14. Distribution, concentration and percentage of surface features $\left(\mathrm{Zn}_{3 \mathrm{C}}, \mathrm{OH}, \mathrm{V}_{\mathrm{o}}\right.$ surface $)$ of NMR detected species on various $\mathrm{ZnO}$ facets of plate (top) and rod (bottom). The concentrations of $\mathrm{Zn}_{4 \mathrm{C}}$ and [O] were not included. Reproduced with permission. ${ }^{[60]}$ Copyright 2016, American Chemical Society.

The quantification and the distribution of various surface features $\left(\mathrm{Zn}_{3 \mathrm{C}}, \mathrm{OH}, \mathrm{V}_{\mathrm{o}}\right.$ surface $)$ on different facets of $\mathrm{ZnO}$ plate and rod at $300 \mathrm{~K}$ are summarized in Figure 14. As like XRD giving structural information on a crystal, it is demonstrated that this surface-fingerprint technique not only provides qualitative (chemical shift) but also quantitative (peak intensity) information on the concentration and distribution of cations and anions, oxygen vacancies and hydroxyl groups on various facets from a single deconvoluted ${ }^{31}$ P NMR spectrum. ${ }^{[60]}$
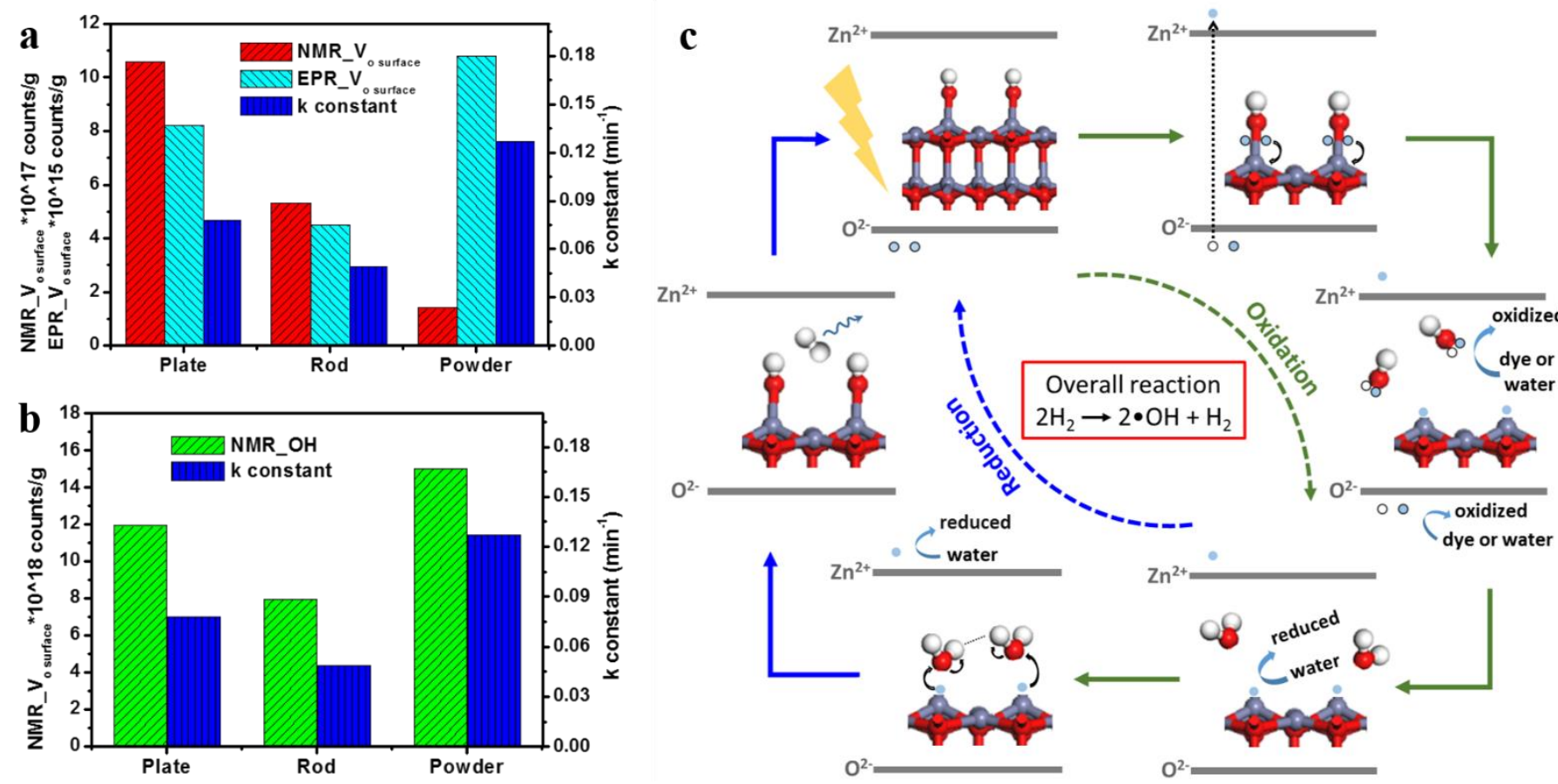

Figure 15. Comparison of (a) $V_{o}$ surface concentration derived by using EPR and NMR spectroscopy and (b) 
$\mathrm{OH}$ concentration derived by using NMR spectroscopy with photocatalytic k constant of ZnO plates, rods, and powder. (c) Schematic illustration of the photocatalytic decomposition of methylene blue dye over $\mathrm{ZnO}$ nanoparticles by $\cdot \mathrm{OH}$ radical in water generated from surface $\mathrm{OH}$. Reproduced with permission. ${ }^{[59]}$ Copyright 2017, Wiley.

Later, Peng et al. further compared this probe-molecule-assisted NMR with both PL and EPR as a characterization tool in facet-specific photocatalysis. ${ }^{[59]}$ From their study, the commonly used PL and EPR spectroscopy techniques were unable to provide a quantitative assessment of the $\mathrm{V}_{\mathrm{o}}$ surface relevant to the facet-dependent activities of oxide nanoparticles. Particularly, PL spectroscopy fails to deliver precise $\mathrm{V}_{\mathrm{o}}$ surface with interferences from bulk defects. Similarly, for EPR, the signal at $\mathrm{g}=2.00$ that originates from trapped unpaired electrons does not necessarily correlate to the $\mathrm{V}_{\mathrm{o}}$ surface concentration because of the equilibrium limitation and there are significant contributions from low-coordinate sites (e.g. surface steps, corners...etc.) from polycrystalline powder particles. ${ }^{[59]}$ The photocatalytic decompositions of methylene blue $(\mathrm{MB})$ in the presence of plates, rods, and powder were carried out to evaluate the facet-dependent catalytic activities. The $\mathrm{k}$ constants for the photocatalytic decompositions were compared with $\mathrm{V}_{\mathrm{o}}$ surface concentrations derived from the EPR and NMR spectra (Figure 15a). Although the apparently higher rate constant of the plates $\left(\mathrm{k}=0.07793 \mathrm{~min}^{-1}\right)$ and the lower rate constant of the rods $\left(\mathrm{k}=0.04869 \mathrm{~min}^{-1}\right)$ can be correlated clearly with the $\mathrm{V}_{\mathrm{o} \text { surface }}$ concentrations derived by both techniques, the polycrystalline powder with the lowest $\mathrm{V}_{\mathrm{o} \text { surface }}$ concentration/polar surface by NMR in the figure shows an unaccountable highest reaction rate constant $\left(\mathrm{k}=0.127 \mathrm{~min}^{-1}\right)$. In addition, the EPR signal originated from trapped unpaired electrons on the surface (i.e. $\mathrm{g}=2.00$ ) cannot be used to attribute to $\mathrm{V}_{\mathrm{o} \text { surface }}$ sites. ${ }^{[59]}$ Carter et al. showed that the $\mathrm{O}_{2}{ }^{-}\left(\mathrm{V}_{\mathrm{o} \text { surface }}\right)$ were the only species exhibiting photochemical reactivity as compared to the other EPR active adsorbed species at surface non-vacancy sites. ${ }^{[69,70]}$ Instead, Peng et al. found the concentration of surface hydroxyl groups shown by using NMR display the same as the order of rate constants (Figure 15b). Accordingly, they linked the photocatalytic $\mathrm{OH}$ radical generation in water from $\mathrm{ZnO}$ NPs directly to their surface $\mathrm{OH}$ groups. As shown in Figure 15c, the $\mathrm{Zn}-\mathrm{OH}$ bond can be photoactivated by electron transfer to $\mathrm{Zn}^{2+}$ to generate an $\mathrm{OH}$ radical. This $\mathrm{OH}$ radical then reacts with water to generate more radicals and can oxidize methylene blue directly. As water is the solvent in this system, the $\mathrm{OH}$ group can be regenerated readily if these photogenerated conduction band electrons on $\mathrm{Zn}$ catalyzes the decomposition of water molecule accompanied with the generation of $\mathrm{H}_{2}{ }^{[59]}$ 
5. Facet-dependent photocatalysis of anatase $\mathrm{TiO}_{2} \mathrm{NPs}$ and their characterization.

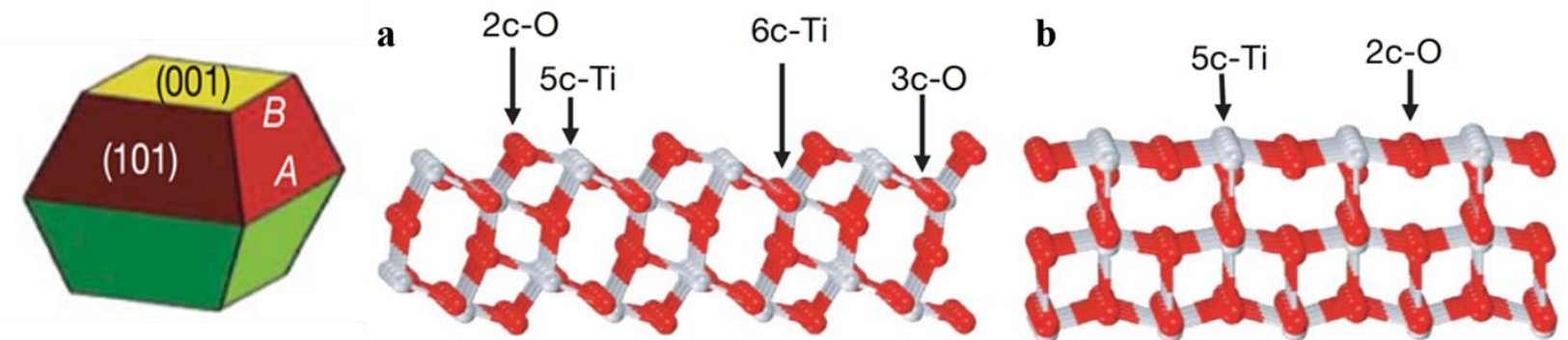

Figure 16. Schematic illustration of the truncated octahedral bipyramidal anatase $\mathrm{TiO}_{2}$ crystal and the two main crystallographic exposed planes (a) (101) and (b) (001). The side lengths labeled A and B are used to define the degree of truncation (B/A) and to estimate the percentage of (001) facets. Reproduced with permission. ${ }^{[52]}$ Copyright 2008 Nature Publishing Group.

Titanium dioxide $\left(\mathrm{TiO}_{2}\right)$ has been the most intensively investigated binary transition metal oxide due to the wide range of its applications for environmental and energy-related areas, especially for (photo)catalysis, dye-sensitized solar cells (DSSCs), lithium ion batteries, sensors, catalyst supports, etc. The unique physical and chemical properties of $\mathrm{TiO}_{2}$ crystals are affected not only by the intrinsic electronic structure, but also by their size, shape and surface properties. ${ }^{[3]}$ In particular, some physicochemical properties such as molecular adsorption, catalytic reactivity and selectivity depend largely on the surface atomic configuration and the percentage of exposed facets. Accordingly, tailoring $\mathrm{TiO}_{2}$ crystals exposed with specific facets have been the hottest research topic since the past decades. ${ }^{[71-74]}$ The truncated octahedral bipyramid anatase $\mathrm{TiO}_{2}$ crystal with eight (101) facets and two (001) facets (Figure 16) is the most common shape observed in nature. According to Wulff construction, the degree of truncation $(\mathrm{B} / \mathrm{A})$ in nature is in the order of $0.3 \sim 0.4$, giving less than $10 \%$ of the exposed $(001)$ facets. ${ }^{[75]}$ Even though various preparation have been attempted, most synthetic anatase $\mathrm{TiO}_{2}$ crystals are mostly covered with thermodynamically more stable (101) facets due to its lower surface free energy $\left(0.44 \mathrm{~J} / \mathrm{m}^{2}\right)$ rather than the reactive high-energy (001) facets $(0.90$ $\left.\mathrm{J} / \mathrm{m}^{2}\right)^{\left[{ }^{[75]}\right.}$ 


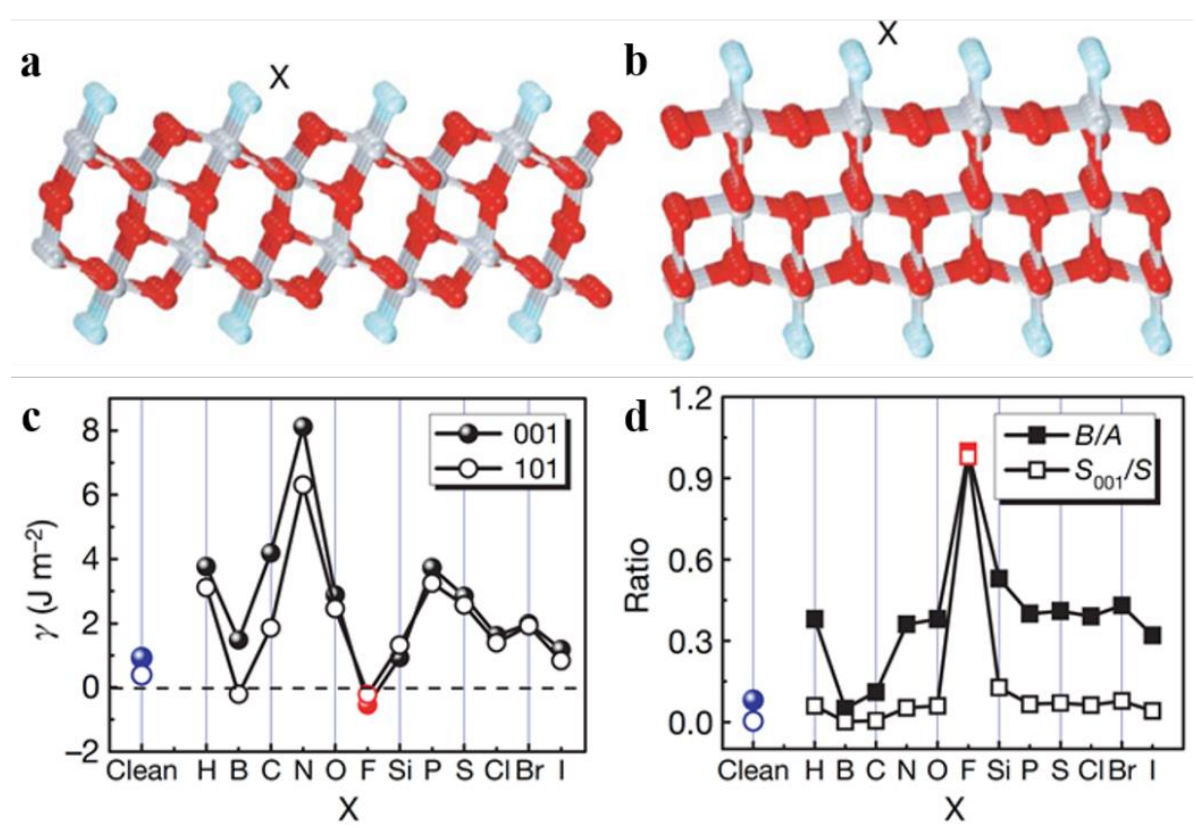

Figure 17. Unrelaxed (a) (101) and (b) (001) surfaces surrounded by adsorbate $X$ atoms. (c) Calculated energies of the (001) and (101) surfaces surrounded by X atoms. (d) Plots of the optimized value of B/A and percentage of (001) facets for anatase single crystals with various adsorbate atoms X. Reproduced with permission. ${ }^{[76]}$ Copyright 2008 Nature Publishing Group.

To synthesize anatase $\mathrm{TiO}_{2}$ crystals with dominant higher energy (001) facets, the crystal growth should to be confined within the kinetically controlled regime under non-equilibrium conditions. Yang et al. theoretically investigated the effects of 12 non-metallic atoms $(\mathrm{X})$ as $\mathrm{SDS}(\mathrm{X}=\mathrm{H}, \mathrm{B}, \mathrm{C}, \mathrm{N}, \mathrm{O}, \mathrm{F}, \mathrm{Si}, \mathrm{P}, \mathrm{S}, \mathrm{Cl}$, $\mathrm{Br}$, or I) on changing the surface energies of anatase (001) and (101) surfaces. ${ }^{[76]}$ As shown in Figure 17a-d, the adsorption of $\mathrm{F}$ reverses the relative order of surface energy and thus the stability of (101) and (001) surfaces, compared to the clean ones. As the growth rate of a crystal facet depends on the surface energy (high-energy facets grow more quickly than low energy facets): this indicates that the formation of F-stabilized (001) facet is now preferable during the particle preparation (Figure 17d). This prediction has been validated firstly by Yang et al. in their synthesis of micro-sized anatase $\mathrm{TiO}_{2}$ with $47 \%$ (001) facet with the addition of HF during hydrothermal preparation (Figure 18a). ${ }^{[76]}$ Later, Han et al. successfully prepared nano-sized anatase crystal with almost doubled the coverage of (001) facets (up to 89\%) by using tetrabutyl titanate, $\mathrm{Ti}(\mathrm{OBu})_{4}$, instead of titanium tetrafluoride $\left(\mathrm{TiF}_{4}\right)$ as precursor (Figure 18b). ${ }^{[77]}$ Since then, using fluoride as SDS to obtain $\mathrm{TiO}_{2}$ nanocrystals with different percentage of exposed (001) facet (by tuning $\mathrm{HF}$ concentration) (Figure 18c) for the investigation of facet activity has been well-documented ${ }^{[71-74]}$ in photocatalysis $^{[77-90]}$, methanol conversion ${ }^{[91]}$, solar cell ${ }^{[92-95]}$ and lithium battery ${ }^{[96,97]}$, etc. 

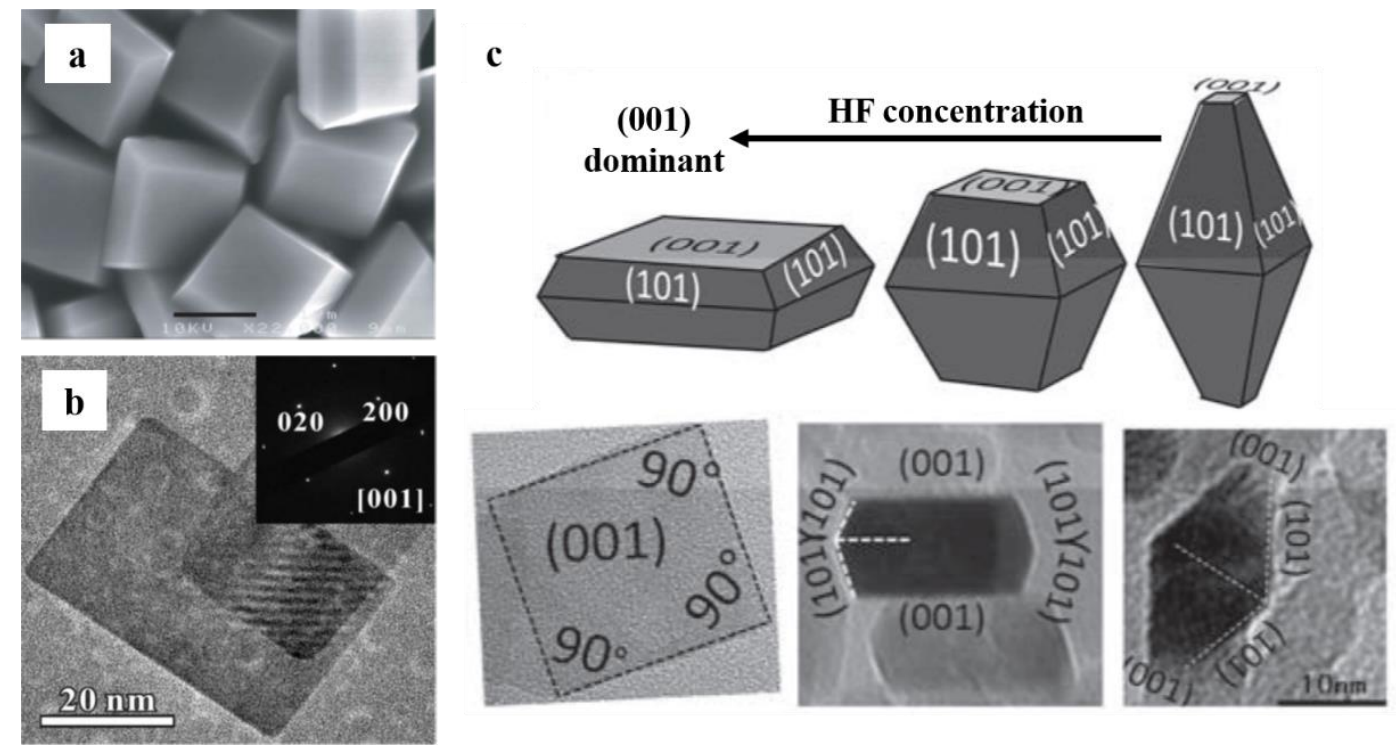

Figure 18. (a) SEM image of anatase $\mathrm{TiO}_{2}$ microsheets with $47 \%$ (001) facet (scale bar: $1 \mu \mathrm{m}$ ). Reproduced with permission. ${ }^{[76]}$ Copyright 2008 Nature Publishing Group. (b) TEM image of anatase $\mathrm{TiO}_{2}$ nanosheet with $89 \%$ (001) facet. Reproduced with permission. ${ }^{[77]}$ Copyright 2009, American Chemical Society. (c) Schematic of the correlation between HF concentration and corresponding products with different percentages of exposed (001) facets. Reproduced with permission. ${ }^{[93]}$ Copyright 2011, Wiley.

In general, the removal of SDS (e.g. fluorine in this case) after the shape control of particle is required to avoid its interfering to the interested properties on facet-activity. Particularly, in the field of heterogeneous catalysis involving the bonds breakage and formation at the catalyst surface is closely associated with the coordination environment of surface active atoms. From the literature, the surface attached F (i.e. F-Ti) can be removed by a simple post-treatment via either calcination at $600^{\circ} \mathrm{C}^{[76,78-80,83,85,87,90-95]}$ or ion exchange with aqueous $\mathrm{NaOH}^{[77,79,81-83,88,89]}$ to obtain a so-called "clean surface". However, by adopting different removal methods, diverse results have been obtained in literature in various applications which have created different interpretations and frequently disagreements amongst researchers ${ }^{[77-97]}$. Here, we summarize corresponding photocatalysis cases in Table 1 . Notice that all the cases in Table 1 are the anatase $\mathrm{TiO}_{2}$ NPs prepared using fluorine as SDS for the morphology control. Accordingly, the $\mathrm{NaOH}$ treated (001) facet (i.e. Na-(001)) showed higher activity in the photocatalytic degradation of methyl orange (MO) dye than the F-capped (001) facet (i.e. F-(001) $)^{[77]}$, while an opposite result was obtained for the same reaction by the other research group $^{[79]}$ (Table 1). Moreover, the environment to carry out photocatalysis seems to play a key factor. The treated $\mathrm{TiO}_{2}$ (i.e. $\mathrm{Cal}-\mathrm{TiO}_{2}$ and $\mathrm{Na}-\mathrm{TiO}_{2}$ ) gave higher photodegradation activity than F-stabilized $\mathrm{TiO}_{2}$ toward methyl orange $(\mathrm{MO})^{[77]}$ and methylene blue $(\mathrm{MB})^{[79]}$ in aqueous phase, while F-stabilized $\mathrm{TiO}_{2}$ showed higher activity for the degradation of acetone ${ }^{[83]}$ and acetaldehyde ${ }^{[84]}$ in air (Table 1). On the other hand, both $\mathrm{NaOH}$ and calcination treatments were reported to increase the facet activity of (101) in 
photocatalytic hydrogen evolution ${ }^{[80,81]}$, while the $\mathrm{NaOH}$ treatment was concluded to lower the facet activity of (001) in this reaction by others (Table 1$)^{[81,82]}$.

Table 1. Facet-controlled F-capped $\mathrm{TiO}_{2}$ NPs treated with various post-treatments (Na: $\mathrm{NaOH}$ wash; Cal: calcination) and their corresponding activities/mechanisms. F-(001) represents as-prepared F-stabilized $\mathrm{TiO}_{2}$ $\mathrm{NP}$ with preferential exposed (001) facet; Na/Cal-(001) represents F-(001) post-treated with $\mathrm{NaOH}$ wash/calcination for the removal of surface fluorine.

\begin{tabular}{|c|c|c|c|c|c|c|}
\hline $\begin{array}{l}\text { Metal } \\
\text { oxide }\end{array}$ & $\begin{array}{l}\text { Crystal } \\
\text { phase }\end{array}$ & $\begin{array}{c}\text { Post- } \\
\text { treatment }\end{array}$ & Photocatalytic reaction & Activity & Proposed mechanism & Ref. \\
\hline $\mathrm{TiO}_{2}$ & Anatase & $\mathrm{NaOH}$ wash & Degradation of MO & $\mathrm{Na}-(001)>\mathrm{F}-(001)$ & Density of unsaturated $\mathrm{Ti}$ & 77 \\
\hline $\mathrm{TiO}_{2}$ & Anatase & Calcination & $\begin{array}{l}\text { Hydroxyl radical } \\
\text { production }\end{array}$ & Cal- $(001)>$ F-(001) & Density of unsaturated $\mathrm{Ti}$ & 78 \\
\hline $\mathrm{TiO}_{2}$ & Anatase & $\begin{array}{l}\mathrm{NaOH} \text { wash/ } \\
\text { Calcination }\end{array}$ & $\begin{array}{l}\text { Degradation of } \mathrm{MO} \& \\
\mathrm{MB}\end{array}$ & $\begin{array}{l}\text { MO: F- }(001)>\mathrm{Na}-(001) \sim \mathrm{Cal}-(001) \\
\text { MB: } \mathrm{Na}-(001)>\mathrm{Cal}-(001)>\mathrm{F}-(001)\end{array}$ & Selective dye adsorption & 79 \\
\hline $\mathrm{TiO}_{2}$ & Anatase & Calcination & $\begin{array}{c}\text { Hydroxyl radical } \\
\text { production } \\
/ \mathrm{H}_{2} \text { evolution from water }\end{array}$ & $\begin{aligned} \mathrm{Cal}-(010) & >\mathrm{Cal}-(101)>\mathrm{Cal}-(001)> \\
\mathrm{F}-(010) & \sim \mathrm{F}-(101) \sim \mathrm{F}-(001)\end{aligned}$ & $\begin{array}{l}\text { Density of unsaturated } \mathrm{Ti} \\
\text { /Band structures }\end{array}$ & 80 \\
\hline $\mathrm{TiO}_{2}$ & Anatase & $\mathrm{NaOH}$ wash & $\mathrm{H}_{2}$ evolution from water & $\begin{array}{c}\mathrm{Na}-(101)>\mathrm{F}-(101)>\mathrm{F}-(001)>\mathrm{Na}- \\
(001)\end{array}$ & Density of unsaturated $\mathrm{Ti}$ & 81 \\
\hline $\mathrm{TiO}_{2}$ & Anatase & $\mathrm{NaOH}$ wash & $\mathrm{H}_{2}$ evolution from water & $\mathrm{F}-(001)>\mathrm{Na}-(001) \sim \mathrm{TiO}_{2}$ & $\begin{array}{l}\text { Enhanced charge separation } \\
\text { (ECS) by residual fluorine }\end{array}$ & 82 \\
\hline $\mathrm{TiO}_{2}$ & Anatase & $\begin{array}{l}\mathrm{NaOH} \text { wash/ } \\
\text { Calcination }\end{array}$ & Degradation of acetone & $\mathrm{F}-\mathrm{TiO}_{2}>\mathrm{Cal}-\mathrm{TiO}_{2} \sim \mathrm{Na}-\mathrm{TiO}_{2}$ & $\begin{array}{l}\text { Synergetic effect of }(001) \& \\
\text { (101) facets \& ECS }\end{array}$ & 83 \\
\hline $\mathrm{TiO}_{2}$ & Anatase & - & $\begin{array}{l}\text { Degradation of } \\
\text { acetaldehyde }\end{array}$ & $\mathrm{F}-\mathrm{TiO}_{2}>\mathrm{TiO}_{2}$ & $\begin{array}{l}\text { Concentration of surface } \\
\text { hydroxyl group }\end{array}$ & 84 \\
\hline $\mathrm{TiO}_{2}$ & Anatase & Calcination & $\mathrm{CO}_{2}$-reduction & $\begin{array}{l}(001) \text { : oxidation } \\
(101) \text { : reduction }\end{array}$ & $\begin{array}{l}\text { Synergetic effect of }(001) \& \\
\text { (101) facets }\end{array}$ & 87 \\
\hline $\mathrm{TiO}_{2}$ & Anatase & $\begin{array}{l}\mathrm{NaOH} \& \mathrm{HF} \\
\text { wash }\end{array}$ & Degradation of $\mathrm{MB}$ & HF-Na-TiO ${ }_{2}>\mathrm{Na}-\mathrm{TiO}_{2}>\mathrm{TiO}_{2}$ & $\begin{array}{l}\text { Synergetic effect of two } \\
\text { facets \& surface defects }\end{array}$ & 88 \\
\hline $\mathrm{TiO}_{2}$ & Anatase & $\mathrm{NaOH}$ wash & $\begin{array}{l}\text { Degradation of } \\
\text { acetaldehyde and phenol }\end{array}$ & $\begin{array}{l}\text { Increases with surface fluorine } \\
\text { concentration }\end{array}$ & $\begin{array}{l}\text { Enhanced adsorbed oxygen } \\
\text { by residual fluorine }\end{array}$ & 89 \\
\hline $\mathrm{TiO}_{2}$ & Anatase & Calcination & Degradation of MB & $\begin{array}{l}\text { Increases with (001) facet for } \\
\text { particle with the same size }\end{array}$ & Density of unsaturated $\mathrm{Ti}$ & 90 \\
\hline
\end{tabular}

It is widely-accepted that any adsorbed molecule on $\mathrm{TiO}_{2}$ surface can efficiently modulate the surface $\mathrm{Ti}$ chemical state, which can deviate largely from that of clean surface. For example, the attachment of $\mathrm{PO}_{4}{ }^{3-} / \mathrm{SO}_{4}{ }^{2-}$ on the $\mathrm{TiO}_{2}$ surface has been reported to provide extra Bronsted Acid (BA) sites and at the same time to increase Lewis acidity (i.e. decrease electron density of surrounding Ti atoms). ${ }^{[98-101]}$ Also, the chelating of surface $\mathrm{COOH}$-containing dye ${ }^{[102]}$ and electrolyte additive such as 4-t-butylpyridine (TBP) ${ }^{[103]}$ have been found to remarkably improve the solar cell performance due to the shift of Ti d-band edge toward negative potentials by adsorption onto the $\mathrm{TiO}_{2}$ surface. On the other hand, the surface reconstruction of $(001)$ from unstable $(1 \times 1)$ to $(1 \times 4)$ upon $\mathrm{F}$ removal during calcination has been shown to result in significantly different $\mathrm{Ti}$ chemical state. Accordingly, those different interpretations made amongst researchers in Table 1 may be arisen from the trace of residue SDS (e.g. F or $\mathrm{OH}$ ) remained on 
surface $^{[88,89,104]}$ or surface reconstruction of unstable (001) upon its removal during calcination ${ }^{[105-107]}$, both of each affecting the chemical state of exposed titanium. So far, no proper surface tool can characterize the surface Ti chemical state on either reconstructed facet or facet promoted with various adsorbed molecules for the correlation of facet activities.

\subsection{Characterization tools employed to correlate facet photoactivity.}

Table 2. Summary of techniques used for anatase $\mathrm{TiO}_{2} \mathrm{NPs}$ characterization. Notice that references selected in this table all use $\mathrm{F}$ as SDS for their morphology controls (S/TEM: scanning/transmission electron microscopy; XPS: X-ray photoelectron spectroscopy; EPR: electron paramagnetic resonance; UV-Vis: ultraviolet-visible spectroscopy; AES: Auger electron spectroscopy; NMR: nuclear magnetic resonance).

\begin{tabular}{|c|c|c|c|c|c|c|c|c|}
\hline Metal oxide & TEM/SEM & XPS & Raman & EPR & UV-Vis & AES & NMR & Ref. \# \\
\hline Anatase $\mathrm{TiO}_{2}$ & $\checkmark$ & $\checkmark$ & & & & & & 76 \\
\hline Anatase $\mathrm{TiO}_{2}$ & $\checkmark$ & & & & & & & 77 \\
\hline Anatase $\mathrm{TiO}_{2}$ & $\checkmark$ & $\checkmark$ & & & & & & 78 \\
\hline Anatase $\mathrm{TiO}_{2}$ & $\checkmark$ & $\checkmark$ & & & & & & 79 \\
\hline Anatase $\mathrm{TiO}_{2}$ & $\checkmark$ & $\checkmark$ & $\checkmark$ & & $\checkmark$ & & & 80 \\
\hline Anatase $\mathrm{TiO}_{2}$ & $\checkmark$ & $\checkmark$ & & $\checkmark$ & & & & 81 \\
\hline Anatase $\mathrm{TiO}_{2}$ & $\checkmark$ & $\checkmark$ & & & $\checkmark$ & & & 82 \\
\hline Anatase $\mathrm{TiO}_{2}$ & $\checkmark$ & $\checkmark$ & & & & & & 83 \\
\hline Anatase $\mathrm{TiO}_{2}$ & $\checkmark$ & $\checkmark$ & & & & & & 84 \\
\hline Anatase $\mathrm{TiO}_{2}$ & $\checkmark$ & $\checkmark$ & & & $\checkmark$ & & & 87 \\
\hline Anatase $\mathrm{TiO}_{2}$ & $\checkmark$ & $\checkmark$ & & $\checkmark$ & & & & 88 \\
\hline Anatase $\mathrm{TiO}_{2}$ & $\checkmark$ & $\checkmark$ & & & $\checkmark$ & & & 89 \\
\hline Anatase $\mathrm{TiO}_{2}$ & $\checkmark$ & $\checkmark$ & & & $\checkmark$ & & & 90 \\
\hline Anatase $\mathrm{TiO}_{2}$ & $\checkmark$ & $\checkmark$ & $\checkmark$ & $\checkmark$ & $\checkmark$ & $\checkmark$ & $\checkmark$ & 104 \\
\hline
\end{tabular}

The tools nowadays employed in the characterization of surfaces with respect to $\mathrm{TiO}_{2}$ facet-dependent photoactivities are summarized in Table 2. Notice that from this table, we only select references using $\mathrm{F}$ as SDS for the $\mathrm{TiO}_{2}$ morphology control since the surface properties (e.g. the chemical state of cations) can vary with different SDS. As shown in the Table 2, the TEM and SEM are the most adopted techniques to identify the preferential exposed facet of a $\mathrm{TiO}_{2}$ nanocrystal. To further monitor element(s) and their oxidation state(s) on material surface, X-ray photoelectron spectroscopy (XPS) analysis is also carried out. As shown in Figure 19a, as the used HF concentration rises, the $\mathrm{F}_{1 \mathrm{~S}}$ intensity of $\mathrm{TiO}_{2}$ samples increases, while no apparent chemical shift and change of intensity were observed for both $\mathrm{Ti}_{2 \mathrm{P}}$ (Figure $19 \mathrm{~b}$ ) and $\mathrm{O}_{1 \mathrm{~S}}$ 
(Figure 19c). ${ }^{[89]}$ Similar results were also reported, the removal of fluorine by either calcination or $\mathrm{NaOH}$ wash can be evaluated by the intensity of XPS F 1 signal (Figure 19d), while no observed chemical shift and change of intensity for $\mathrm{Ti}_{2 \mathrm{P}}$ (Figure 19e). ${ }^{[104]}$ This might be due to the long electron escaping depth with more than the outmost atomic layer (few atoms depth) from sample, which renders the XPS not a truly surface technique (detection limit $\sim 0.1 \%$ atom). Even though Auger electron spectroscopy (AES) is known to provide higher spatial resolution as the energy of ejected Auger electrons is much sensitive to chemical environment compared to the core-level signals in XPS, no peak shift of $\mathrm{Ti}_{2 \mathrm{P}}$ in the presence of fluorine was observed for five Ti LMM Auger signals (marked by dashed blue line) (Figure 19f). ${ }^{[104]}$ This indicates the change of chemical state of the outmost Ti atom is still averaged out during the collection of Auger electrons from the electron escaping depth.
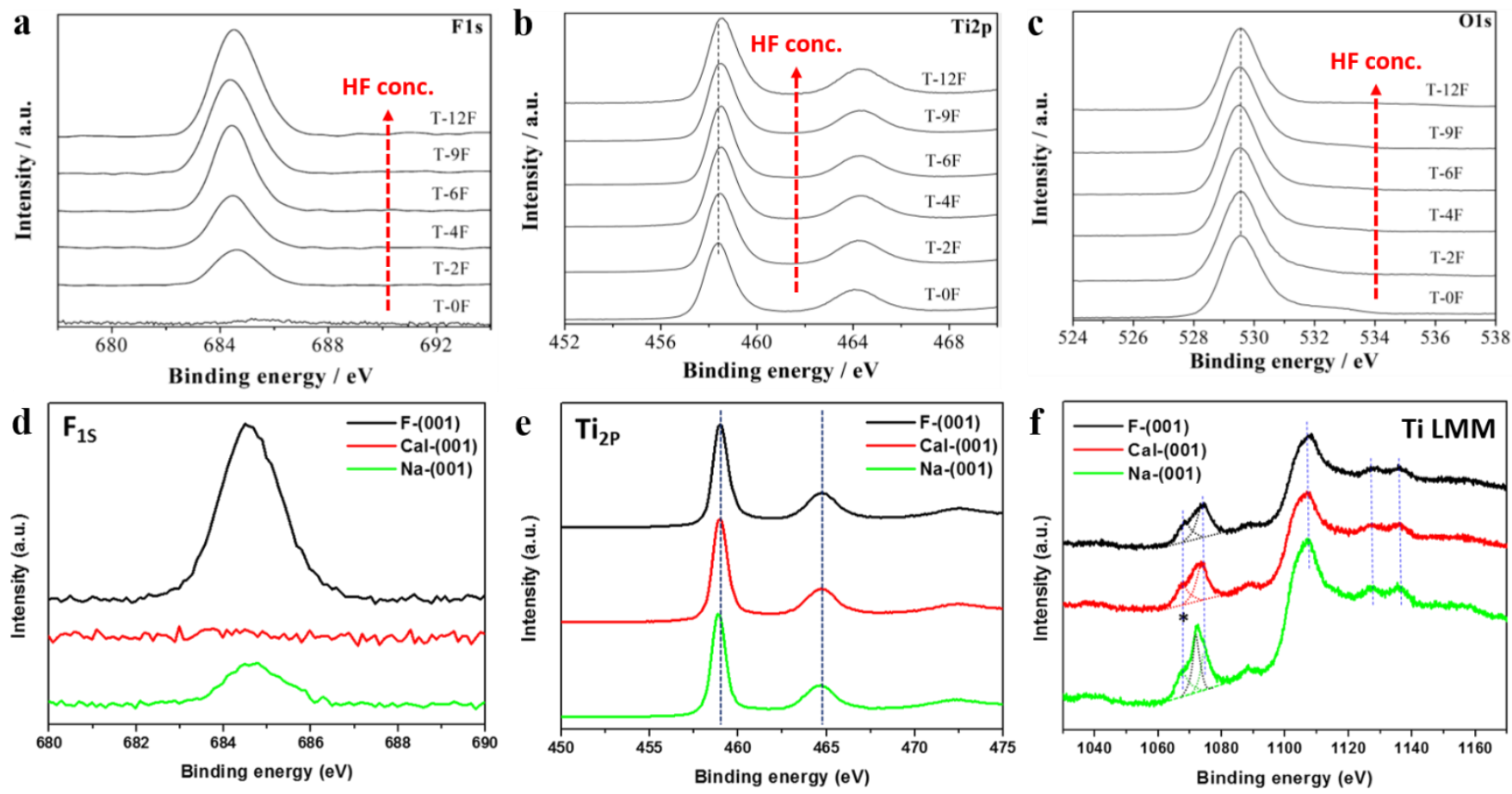

Figure 19. a-c) XPS spectra of (a) $\mathrm{F}_{1 \mathrm{~S}}$, (b) $\mathrm{Ti}_{2 \mathrm{P}}$ and (c) $\mathrm{O}_{1 \mathrm{~S}}$ of $\mathrm{F}$-stabilized $\mathrm{TiO}_{2}$ samples prepared with 0 to $1.2 \mathrm{~mL}$ of $40 \%$ HF. Reproduced with permission. ${ }^{[89]}$ Copyright 2013, American Chemical Society. d-f) XPS spectra of (d) $\mathrm{F}_{1 \mathrm{~S}}$, (e) $\mathrm{Ti}_{2 \mathrm{P}}$ and (f) $\mathrm{Ti}$ LMM (Auger spectra) of F-stabilized (001) $\mathrm{TiO}_{2}$ samples with different post-treatments (Cal: calcination and $\mathrm{Na}$ : $\mathrm{NaOH}$ wash). The peak marked with asterisk "*" in figure (f) is $\mathrm{Na}_{1 \mathrm{~S}}$ signal. Reproduced with permission. ${ }^{[104]}$ Copyright 2017 Nature Publishing Group.

According to the literature ${ }^{[80,108,109]}$ the removal of surface fluorine can also be monitored by Raman spectroscopy. It has been shown that the surface attached fluorine changes both symmetry of Ti-O-Ti and coordination of surface $\mathrm{Ti}$ atom, resulting in the shift of low-frequency $\mathrm{E}_{\mathrm{g}}$ and weakening of $\mathrm{B}_{1 \mathrm{~g}}\left(\mathrm{cf} . \mathrm{A}_{1 \mathrm{~g}}\right)$ after the fluorine removal (Figure 20a). ${ }^{[108]}$ The EPR signal at $\mathrm{g} \sim 2.0$ (unpaired electrons trapped in $\mathrm{V}_{\mathrm{o}}$ via 
adsorbed oxygen species from air $\left(\mathrm{O}_{2}^{-}\right)$) and $\mathrm{g} \sim 1.98$ (unpaired electrons trapped by surface/subsurface paramagnetic $\mathrm{Ti}^{3+}$ center) have been used to evaluate the effect of $\mathrm{F}$ attachment, $\mathrm{NaOH}$ washing and subsequently HF treatment to the surface (Figure 20b). ${ }^{[88]}$ EPR was also adopted by D'Arienzo et al. to investigate the relative contribution of each exposed crystal facet to the photoactivity. ${ }^{[86]}$ The (001) surface was found playing the major role in the photocatalytic process by providing oxidation sites, while the (101) surface was involved as the supplier of reductive sites. This synergetic effect of different facets has been shown effectively to reduce the electron-hole recombination and further enhance their photocatalytic activity. ${ }^{[83,85,84,88]}$ Pan et al. attributed the photocatalytic performance of facet-controlled $\mathrm{TiO}_{2}$ to the band gap size of the exposed facets determined by ultraviolet-visible (UV-vis) spectra together with valence band XPS measurement $(\mathrm{T} 010>\mathrm{T} 101>\mathrm{T} 001)\left(\right.$ Figure 20c). ${ }^{[80]}$ However, the influence of the particle size on band gap of semiconductor was neglected in their work. To demonstrate this, Wu et al. prepared anatase $\mathrm{TiO}_{2}$ single crystals with different percentage of (001) facets in uniform size, as well as anatase $\mathrm{TiO}_{2}$ single crystals with different particles size in same percentage of (001) facets. ${ }^{[90]}$ Their results showed the band gap of anatase $\mathrm{TiO}_{2}$ crystals was nearly independent with the percentage of different reactive facets, while the band gap would be much narrower with smaller particles size. Therefore, the higher photoreactivity of (101) facet than that of (001) facets in Pan's work ${ }^{[80]}$ could be associated with the much smaller particle size of the sample with high percentage of (101) facets.
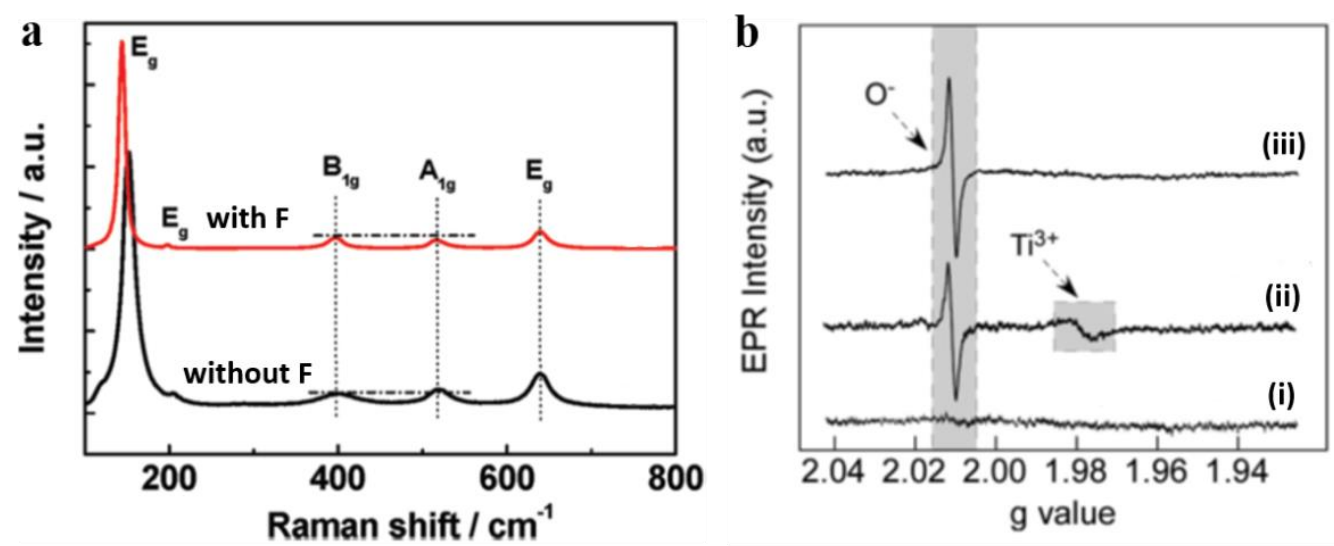

c

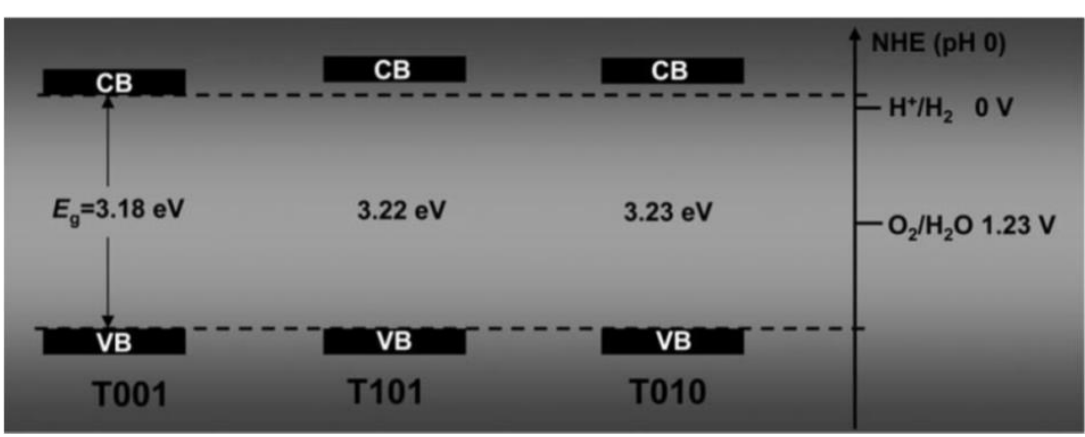

Figure 20. (a) Raman spectra of anatase $\mathrm{TiO}_{2}$ sheets with/without surface F. Reproduced with 
permission. $^{[108]}$ Copyright 2009, American Chemical Society. (b) EPR spectra of (i) as-synthesized F-stabilized $\mathrm{TiO}_{2}$, (ii) after $\mathrm{NaOH}$ wash and subsequently (iii) $\mathrm{HF}$ treatment. Reproduced with permission. ${ }^{\text {[8] }}$ Copyright 2015, American Chemical Society. (c) The determined band-gap size of anatase $\mathrm{TiO}_{2}$ crystals with preferential exposed (001), (101) and (010) facets. Reproduced with permission. ${ }^{[80]}$ Copyright 2011, Wiley.

In addition to band structure, the commonly used criteria to explain the photoactivity of $\mathrm{TiO}_{2}$ facets is the density of surface undercoordinated atoms (Table 1). ${ }^{[77,78,80,81,90]}$ Those literatures have assumed they obtained clean surface after the F removal and attributed the high photoactivity of (001) facet to the $100 \%$ surface unsaturated $\mathrm{Ti}_{5 \mathrm{C}}$ atoms on (001) facets (cf. 50\% for the (101) facets) (Figure 16). However, as shown above, these so called 'surface tools' are used mainly for the proof of F removal (i.e. XPS, Raman, and EPR) or electronic structure (i.e. UV-vis and XPS), they are not truly facet specifics. Accordingly, they provide very limited information on the chemical state of surface features $\left(\mathrm{V}_{\mathrm{o}}, \mathrm{OH}\right.$, cations...etc.) and their distribution among facets, causing difficulty to unambiguously correlate facet-dependent results during the past decades.

\subsection{Treatment-dependent surface features.}

In addition to the limitations from technical aspects mentioned above, different post-treatments adopted during the particle preparation (e.g. calcination at $600^{\circ} \mathrm{C}$ in air $/ 0.1 \mathrm{M} \mathrm{NaOH}$ wash here) further increase the difficulty in surface characterization. Given that each facet possesses distinctive intrinsic energy, it is understandable that both concentration and chemical state of surface features differ from facet to facet after reaching equilibrium with post-treatments. Recently, the calcination removal of fluorine has been shown to cause stacked $\mathrm{TiO}_{2}$ nanosheets along particle [001] crystallographic direction, driven by the minimization of surface energy. ${ }^{[110,111]}$ The surface Ti-F groups can be totally hydrolysed by water during calcination in air at high temperature $\left(600^{\circ} \mathrm{C}\right.$, endothermic reaction) (Figure 21a). In this case, the exposed surfaces of sheet-like anatase $\mathrm{TiO}_{2}$ are also mainly covered by -OH groups. As (001) facet has a higher surface energy than (101) facet, the (001) interfaces between adjacent nanosheets would be eliminated through the condensation of Ti-OH groups (formation of Ti-O-Ti linkages) at high temperature and therefore the (101) facet becomes dominant (Figure 21a). Also, (001) facet has been recently shown to reconstruct from (1 x 1$)$ to a more stable $(1 \mathrm{x} 4)$ surface at high temperature by environmental TEM $^{[106]}$ and in situ STEM ${ }^{[107]}$. Accordingly, the formation of surface Ti-OH, elimination of (001) facet and reconstruction of the rest (001) facet should be 
also included for activity consideration by study using calcination as post-treatment. Comparing to calcine-induced aggregation (Figure 21c), no apparent morphology change can be observed for $\mathrm{NaOH}$ wash at room temperature (Figure 21d) (cf. F-stabilized $\mathrm{TiO}_{2}$, Figure 21e). However, the $\mathrm{NaOH}$ wash has been reported to induce changes in the surface morphology, including step density and point defects. ${ }^{[88]}$ Such changes might have an influence on the photoactivity after the $\mathrm{NaOH}$ wash. Also, in some cases, the $\mathrm{F}$ removal was not complete by $\mathrm{NaOH}$ wash (from XPS $\left.\mathrm{F}_{1 \mathrm{~S}}\right)^{[88,89,104]}$ presumably due to ambient reaction temperature used, while no $\mathrm{F}$ was found in all calcined samples by the XPS $\mathrm{F}_{1 \mathrm{~S}}$ analysis. ${ }^{[76,78-80,83,87,90-95]}$ The surface $\mathrm{F}$ residue (i.e. Ti-F group) has been shown to act as an electron-trapping site but to reduce interfacial electron transfer rates by tightly holding trapped electrons due to the strong electronegativity of the fluorine. ${ }^{[112]}$ Also, different forms of residue $\mathrm{F}$ on $\mathrm{TiO}_{2}$ surface have been found at ambient conditions and water. As shown by Luan et al., the surface of F-residual $\mathrm{TiO}_{2}$ possessed $-\mathrm{Ti}-\mathrm{OH},-\mathrm{Ti}-\mathrm{F}$ and $-\mathrm{Ti}: \mathrm{F}-\mathrm{H}$ groups in the air, while $-\mathrm{Ti}: \mathrm{F}-$ was found from the dissociation of the $-\mathrm{Ti}: \mathrm{F}-\mathrm{H}$ group in water (Figure 21b) ${ }^{\left[{ }^{[8]}\right.}$ They suggested that the $-\mathrm{Ti}: \mathrm{F}-\mathrm{H}$ could greatly enhance $\mathrm{O}_{2}$ adsorption and thus greatly promote the charge separation and the photocatalytic activity. This finding may explain some controversies in the reported literature as different post-treatments (calcination $/ \mathrm{NaOH}$ wash) and reaction conditions (ambient/water) would lead to various - Ti:F-H concentration.

a

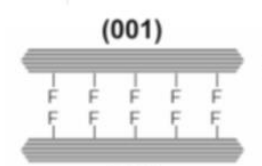

(001)

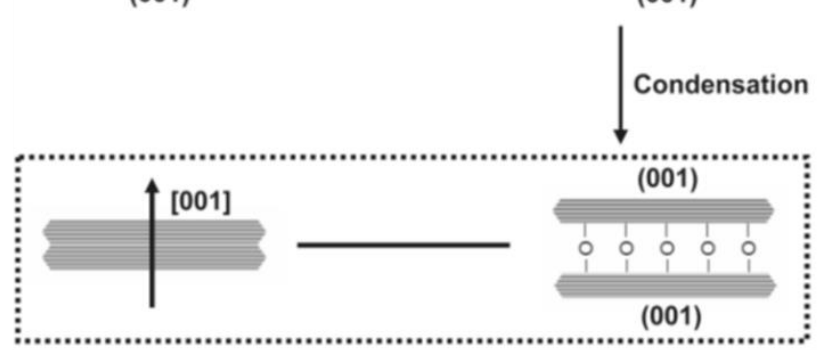

(001) Surface Hydrolysis
of Ti-F groups b

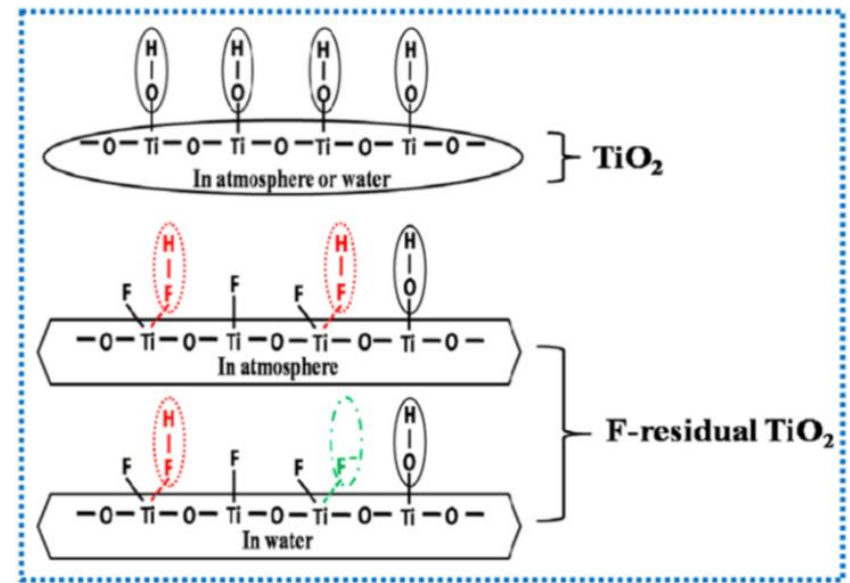

c

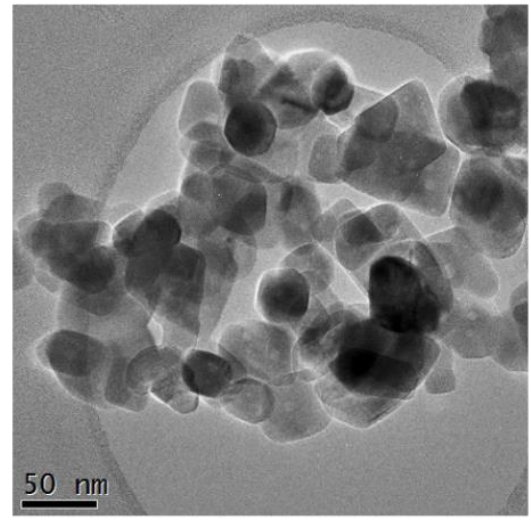

d

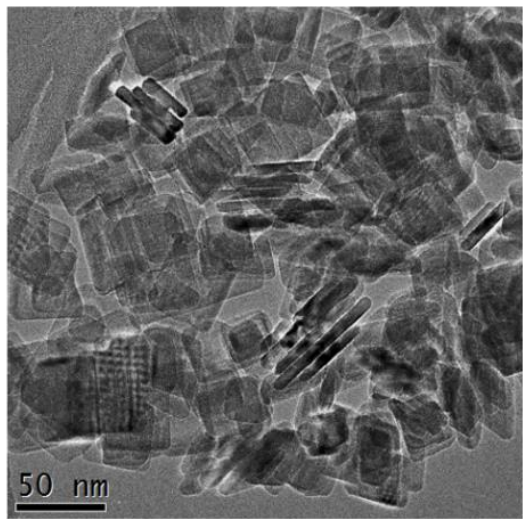

e

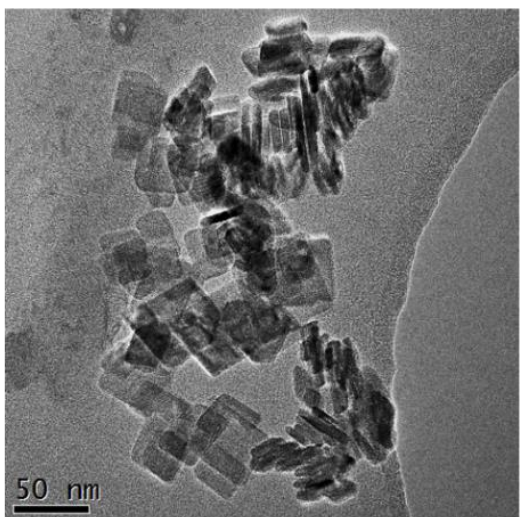


Figure 21. (a) Schematic illustration of the stacked anatase $\mathrm{TiO}_{2}$ nanosheets (aggregation) by calcination treatment. Reproduced with permission. ${ }^{[110]}$ Copyright 2011, American Chemical Society. (b) Surface features schematic of $\mathrm{TiO}_{2}$ and $\mathrm{TiO}_{2}$ with $\mathrm{F}$ residues in atmosphere/water. Reproduced with permission. ${ }^{[89]}$ Copyright 2011, American Chemical Society. c-e) TEM images of (c) calcined, (d) $\mathrm{NaOH}$ washed and (e) as-prepared $\mathrm{TiO}_{2}$ nanosheets. Reproduced with permission. ${ }^{[104]}$ Copyright 2017 Nature Publishing Group.

\subsection{Using probe-assisted NMR as a mapping technique for facets promoted with various surface}

\section{groups.}

Very recently, the power of probe-assisted NMR has been demonstrated not only can differentiate/quantify surface features on various facets ${ }^{[60]}$ but can also further monitor the changes of their chemical states induced by a small quantity of surface adsorbates. ${ }^{[104]}$ Peng et al. have successfully monitored the subtle changes of Ti chemical state induced by surface attached fluorine, sulfate and hydroxyl group on various facets using ${ }^{31} \mathrm{P}$ MAS NMR in combined with TMP as surface probe. ${ }^{[104]}$ As mentioned in Sec. 4.3, this technique is because the nucleophilic sensitive probe molecule, TMP, can form a stable adduct with the exposed $\mathrm{Ti}$ cation (Lewis acid, $\mathrm{LA}$ ) of $\mathrm{TiO}_{2}$ surface, the formation of a surface TMP-Ti complex can be realized by the coordination of the $\mathrm{P}$ atom to the surface Ti cation center. As the stronger interaction between TMP and surface Ti pushes ${ }^{31} \mathrm{P}$ chemical shift $\left(\delta^{31} \mathrm{P}\right)$ toward downfield (i.e. zero ppm), Ti cations on different facets promoted with various surface groups can thus be distinguished by corresponding chemical shifts and analyzed quantitatively.

a

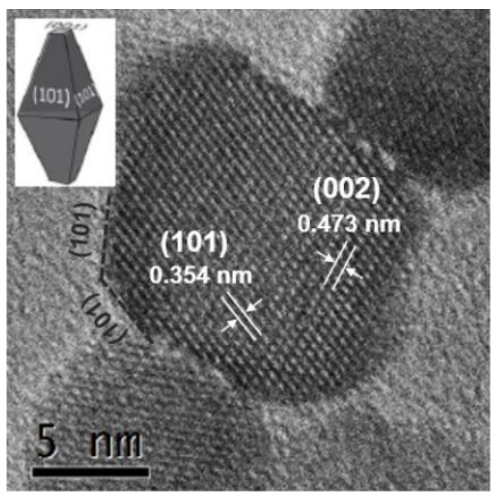

d

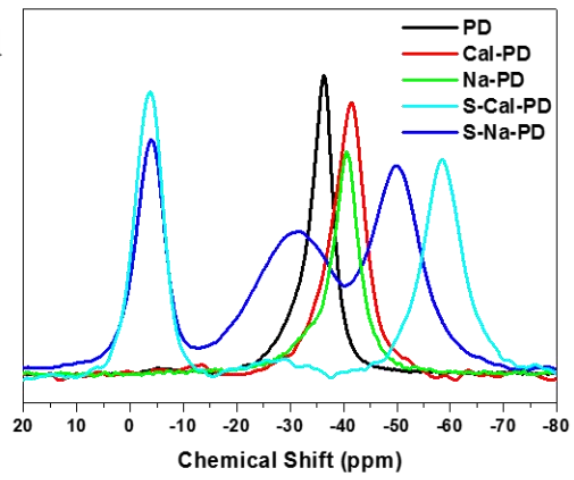

b
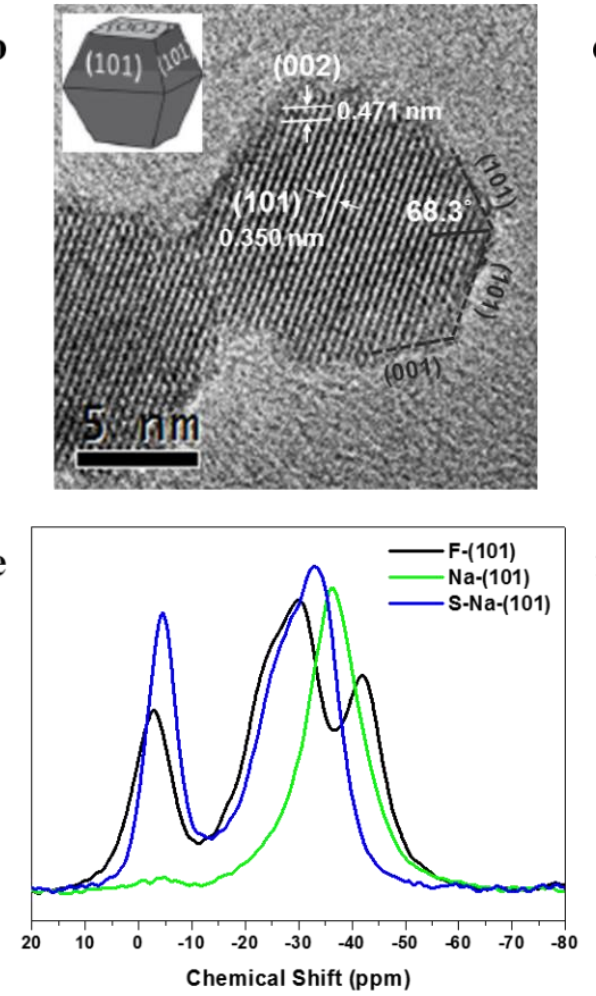

c
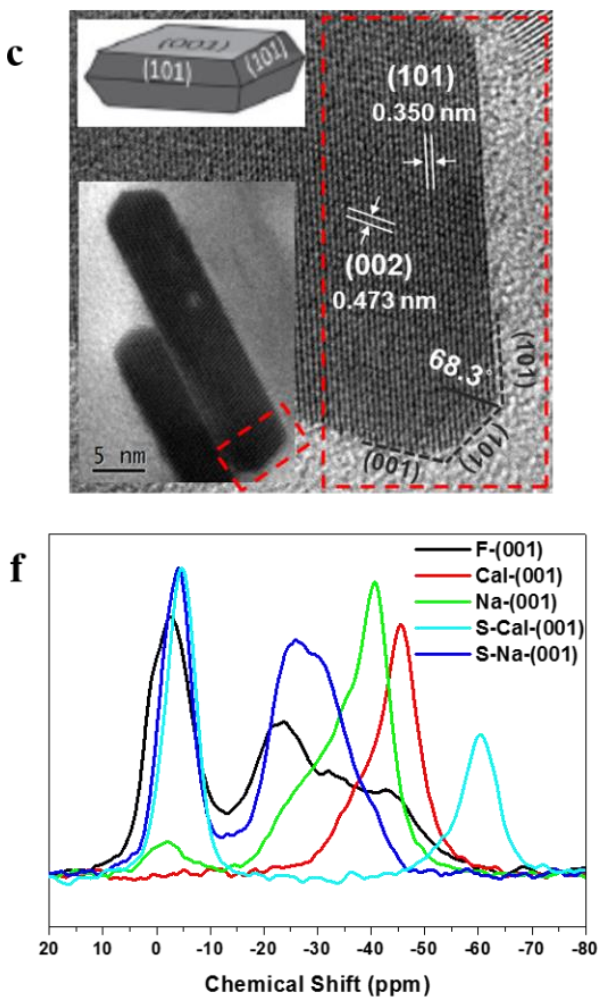

Figure 22. a-c) TEM images of as-prepared (a) powder (PD), (b) F-(101) and (c) F-(001). d-f) ${ }^{31}$ P MAS 
NMR spectra showing the electronic effect (chemical shift) imposed by different adsorbates during sequential treatments/modifications to surface cation on as-prepared (d) powder (PD), (e) F-(101) and (f) F-(001) (black line) with calcination (red line) or $\mathrm{NaOH}$ wash (green line) followed by sulfate modification (pale blue and blue line) (Cal: calcination; $\mathrm{Na}$ : $\mathrm{NaOH}$ wash; $\mathrm{S}$ : sulfation). Reproduced with permission. ${ }^{[104]}$ Copyright 2017 Nature Publishing Group.

To evaluate the potential of this technique, Peng et al. prepared $\mathrm{TiO}_{2}$ powder (PD), F-(101) and F-(001) with the addition of $0 \mathrm{~mL}, 2 \mathrm{~mL}$ and $6 \mathrm{~mL}$ of $50 \mathrm{wt} \% \mathrm{HF}$ (Figure 22a-c). The percentages of exposed (001) facets of powder, F-(101) and F-(001) were estimated to be 10.2\%, 21.1\% and 75.4\%, respectively. Figure 22d-f shows their corresponding ${ }^{31} \mathrm{P}$ MAS NMR spectra before/after calcination/ $\mathrm{NaOH}$ wash and subsequent sulfation. Regardless the physicochemical meaning of each peak, this technique indeed provides extraordinary sensitivity to the chemical states of surface features than traditional surface tools shown above. Its power to distinguish the subtle difference in chemical state of $\mathrm{Ti}_{5 \mathrm{C}}$ atoms makes it feasible to monitor the electronic effect imposed by different adsorbates during sequential treatments/modifications to NP surface cation (Figure 22d-f).
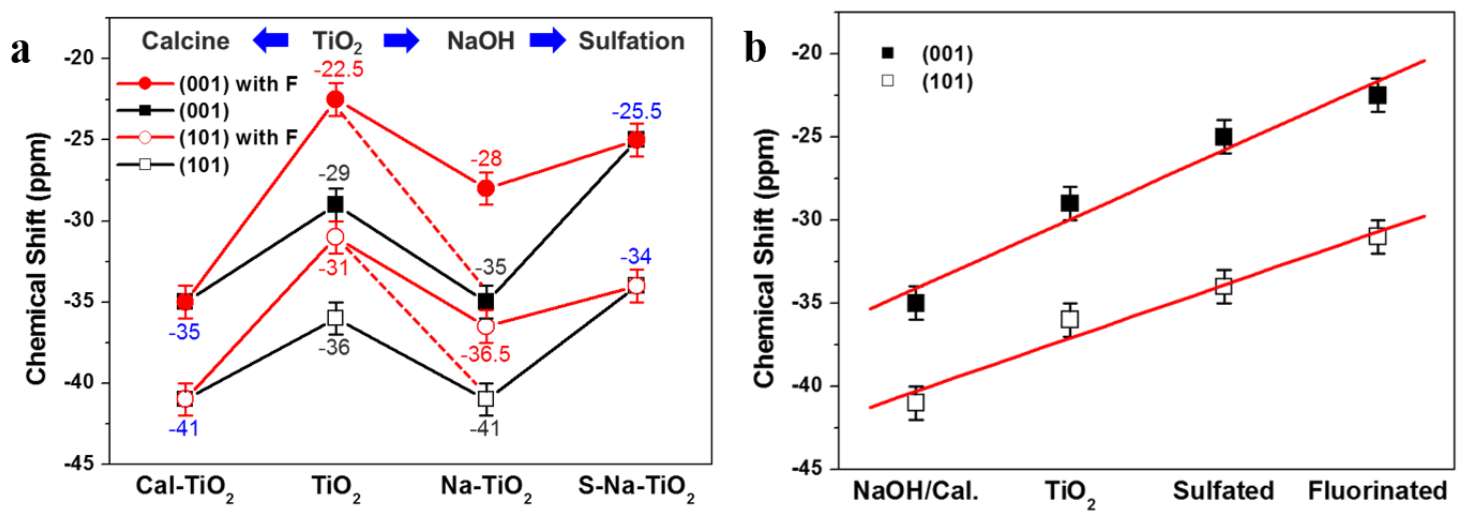

c
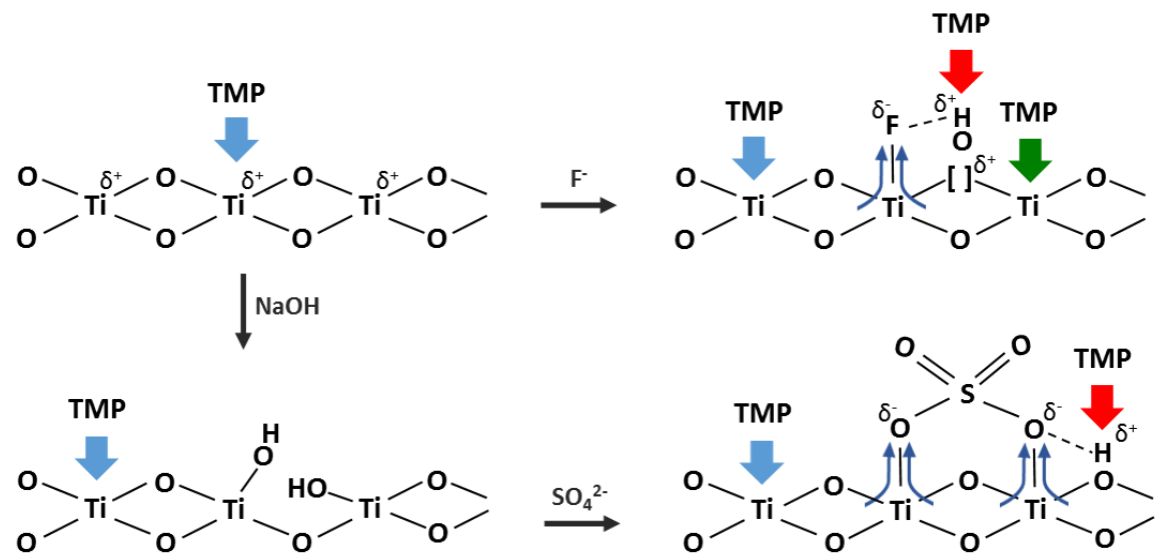

Figure 23. (a) The $\delta^{31} \mathrm{P}$ of TMP-adsorbed $\mathrm{Ti}_{5 \mathrm{C}}$ on (001) and (101) facets w/o surface $\mathrm{F}$ after post treatments (calcination/ $\mathrm{NaOH}$ wash) and subsequently sulfate modifications. (b) The summary of $\delta^{31} \mathrm{P}$ of TMP-adsorbed $\mathrm{Ti}_{5 \mathrm{C}}$ on (001) and (101) facets with different treatments (calcination/ $\mathrm{NaOH}$ wash) and modification (sulfate/fluorine). (c) Illustration of interaction between TMP and surface features on $\mathrm{TiO}_{2}$ facet with various treatments/modifications (blue arrow: Ti ${ }_{5 \mathrm{C}}$; red arrow: BA site; green arrow: oxygen vacancy). Reproduced with permission. ${ }^{[104]}$ Copyright 2017 Nature Publishing Group. 
According to that study, the adsorption of $\mathrm{TMP}$ on $\mathrm{Ti}_{5 \mathrm{C}}$ of higher energy (001) facet gives lower chemical shift value $(-29 \mathrm{ppm})$ than that of the more stable (101) facet (-36 ppm) (Figure 23a). The F-stabilized (001) and (101) facets can clearly enhance the LA strength of $\mathrm{Ti}_{5 \mathrm{C}}$, shifting them to $-22.5 \mathrm{ppm}$ and -31 ppm, respectively (Figure 23a). The post-calcination (in air) treatment (i.e. $\mathrm{Cal}-\mathrm{TiO}_{2}$ ) can efficiently replace $\mathrm{F}$ by much weaker surface $\mathrm{OH}$ group rendering the upshifts to $-35 \mathrm{ppm}$ for (001) facet and $-41 \mathrm{ppm}$ for (101) facet, respectively (Figure 23a). The same chemical shifts for both facets were obtained by $\mathrm{NaOH}$ wash at room temperature (i.e. $\mathrm{Na}^{-\mathrm{TiO}_{2}}$ ). However, the $\mathrm{NaOH}$ wash in most cases may not be sufficient to remove all surface $\mathrm{F}$ content from the $\mathrm{TiO}_{2}$. If $\mathrm{F}$ is retained together with the $\mathrm{OH}$ can give two extra $\delta^{31} \mathrm{P}$ values at $-28 \mathrm{ppm}$ and $-36.5 \mathrm{ppm}$, respectively (Figure 23a). This may explain the diverse results have been obtained among researchers by adopting calcination or $\mathrm{NaOH}$ wash to remove surface $\mathrm{F}$. Upon sulfation after surface $\mathrm{F}$ is removed, $-25.5 \mathrm{ppm}$ from sulfation of (001) and $-34 \mathrm{ppm}$ of (101) facets can be tuned (Figure 23a). As shown in Figure 23b, the highly sensitive $\delta^{31} \mathrm{P}$ value towards increasing electron withdrawing ability of surface additives to the surface $\mathrm{Ti}^{4+}$ from $\mathrm{OH}<-\mathrm{O}-<\mathrm{SO}_{4}<\mathrm{F}$ can be distinguished on $\mathrm{TiO}_{2}$ facets. The anticipated molecular interactions between TMP and surface features on $\mathrm{TiO}_{2}$ with various treatments/modifications are illustrated in Figure 23c. As the strength and concentration of BA and LA can be obtained by this technique, they can be used to elucidate the mechanism of photocatalysis. According to their result, the concentration of LA sites is the rate-determining step for photo-catalytic dye degradation in static solution, while the adsorption dynamic of molecules in flowing air is very different from that of dye in static water that can only be efficiently adsorbed from the gas phase by the stronger LA site. ${ }^{[104]}$ This result may explain the different activity found previously that of the treated $\mathrm{TiO}_{2}$ (i.e. $\mathrm{Cal}_{-} \mathrm{TiO}_{2}$ and $\mathrm{Na}-\mathrm{TiO}_{2}$ ) which gives higher photodegradation activity than F-stabilized $\mathrm{TiO}_{2}$ toward methyl orange $(\mathrm{MO})^{[77]}$ and methylene blue $(\mathrm{MB})^{[79]}$ in aqueous medium. Whereas, F-stabilized $\mathrm{TiO}_{2}$ shows higher activity for the degradation of acetone ${ }^{[83]}$ and acetaldehyde ${ }^{[84]}$ in a flowing stream of air (Table 1).

\section{Conclusions and perspectives}

Undoubtedly, the development of semiconductive transition metal oxides with tailored facets has been one of the most significant events in environment and electronics related photocatalytic research in the past decades. Ideally, the continuing development of these catalysts with facet-dependent activity would not only 
deepen the understanding of the structural sensitivity of such catalysts, by correlating their reaction performance with their dominant crystal facet, but also bridge the gap between the model catalysts used in surface science and the real catalysts found in practical applications. Often, however, comparison of such facet-dependence is limited to individual studies, as the processing and post-treatments used in the preparation of catalysts or later removal of adsorbates (e.g. surfactant employed in facet control) varies from group to group. In most cases, those so-called "clean surfaces" were not so clean as they claimed since each facet possesses distinctive intrinsic energy that both concentration and chemical state of surface active features $\left(\mathrm{V}_{\mathrm{o}}, \mathrm{OH}\right.$, cations...etc.) should differ from facet to facet after reaching equilibrium with post-treatments. As residue adsorbates have been shown remain on the facets and influence the adsorption of reactant molecules and the chemical states of surface active features, this forms an obstacle for reliably evaluating the facet-dependent photocatalytic performance. Moreover, from characterization side, traditional surface techniques such as PL, EPR, Raman, XPS and AES are collecting data from the bulk of particle while not from the specific facets. Even though XPS and AES have been regarded as the most sensitive technique to surface atomic chemical state, it fails to give any chemical shift of surface features in the presence of adsorbates. Accordingly, these traditional techniques provide very limited information on the chemical state of surface active features and their distribution among facets, causing difficulty to unambiguously correlate facet-dependent results and thus always lead to different interpretations amongst researchers during the past decades. Until very recently, the probe-molecule-assisted NMR has been demonstrated to allow the differentiation of surface active features from various facets and unprecedentedly provide both qualitative (chemical shift) and quantitative (peak intensity) information on their concentration and distribution. This advance in facet-specific characterization technique gives new insights to resolve the current debates and confusions in some facet-dependent properties in literature. In combining with traditional techniques, this technique can open up a new route for the understanding of photocatalytic mechanisms. Overall, the development of semiconductive transition metal oxides with tailored facets and surface characterization techniques should keep pace with each other in order to unambiguously correlate the facet-dependent surface feature with corresponding performance. 


\section{References}

[1] M. N. Chong, B. Jin, C. W. K. Chow, C. Saint, Water Research 44 (2010) 2997-3027

[2] K. M. Lee, C. W. Lai, K. S. Ngai, J. C. Juan, Water Research 88 (2016) 428-448.

[3] J. Schneider, M. Matsuoka, M. Takeuchi, J. Zhang, Y. Horiuchi, M. Anpo, D. W. Bahnemann, Chem. Rev. 114 (2014) 9919-9986.

[4] B.-G. Zhai, Y. M. Huang, Optoelectron. Mater. 1 (2016) 22-36.

[5] S. Bai, L. Wang, Z. Li, Y. Xiong, Adv. Sci. 4 (2017) 1600216.

[6] H. H. Chen, C. E. Nanayakkara, V. H. Grassian, Chem. Rev. 112 (2012) 5919-5948.

[7] X. B. Chen, S. H. Shen, L. J. Guo, S. S. Mao, Chem. Rev. 110 (2010) 6503-6570.

[8] X. Chang, T. Wang, J. Gong, Energy Environ. Sci. 9 (2016) 2177-2196.

[9] D. Gust, T. A. Moore, A. L. Moore, Acc. Chem. Res. 42 (2009) 1890-1898.

[10] I. Rossetti, ISRN Chem. Eng. 2012 (2012) 1.

[11] M. Gratzel, Nature 414 (2001) 338-344.

[12] Z. R. Tian, J. A. Voigt, J. Liu, B. Mckenzie, M. J. Mcdermott, M. A. Rodriguez, H. Konishi, H. Xu, Nat Mater. 2 (2003) 821-826.

[13] S. G. Kumar, K. S. R. K. Rao, RSC Adv. 5 (2015) 3306-3351.

[14] X. B. Chen, L. Liu, P. Y. Yu and S. S. Mao, Science 331 (2011) 746-750.

[15] M. Setvin, U. Aschauer, P. Scheiber, Y. F. Li, W. Y. Hou, M. Schmid, A. Selloni, U. Diebold, Science 341 (2013) 988-991.

[16] X. Q. Chen, J. H. Ye, S. X. Ouyang, T. Kako, Z. S. Li, Z. G. Zou, ACS Nano 5 (2011) 4310-4318.

[17] G. C. Xi, J. H. Ye, Q. Ma, N. Su, H. Bai, C. Wang, J. Am. Chem. Soc. 134 (2012) 6508-6511.

[18] Z. Han, F. Qiu, R. Eisenberg, P. L. Holland, T. D. Krauss, Science 338 (2012) 1321-1324.

[19] T. Simon, N. Bouchonville, M. J. Berr, A. Vaneski, A. Adrovic, D. Volbers, R.Wyrwich, M. Döblinger, A. S. Susha, A. L. Rogach, F. Jäckel, J. K. Stolarczyk, J. Feldmann, Nat Mater. 13 (2014) 1013-1018.

[20] Y. Li, Y.-L. Li, C. M. Araujo, We. Luo, R. Ahuja, Catal. Sci. Technol. 3 (2013) 2214-2220.

[21] W.-J. Ong, L.-L. Tan, Y. H. Ng, S.-T. Yong, S.-P. Chai, Chem. Rev. 116 (2016) 7159-7329.

[22] A. Dhakshinamoorthy, A. M. Asiri, H. García, Angew. Chem. Int. Ed. 55 (2016) 5414-5445.

[23] M. R. Hoffmann, S. T. Martin, W. Choi, D. W. Bahnemann, Chem. Rev. 95 (1995) 95, 69-96.

[24] Ohtani, B. Catalysts 3 (2013) 942-953.

[25] D. Beydoun, R. Amal, G. Low, S. McEvoy, J. Nanopart. Res. 1 (1999) 439-458.

[26] H. S. Fogler, in: Elements of Chemical Reaction Engineering, Chapter 10: Catalysis and Catalytic Reactors. Prentice-Hall PTR Inc, 1991, pp. 581-685.

[27] K. Vinodgopal, P. V. Kamat, J. Phys. Chem. 96 (1992) 5053-5059.

[28] G. Liu, J. C. Yu, G. Q. Lu, H. M. Cheng, Chem. Commun. 47 (2011) 6763-6783.

[29] K. Zhou, Y. Li, Angew. Chem. Int. Ed. 51 (2012) 602-613.

[30] H. Xu, P. Reunchan, S. Ouyang, H. Tong, N. Umezawa, T. Kako, J. Ye, Chem. Mater. 25 (2013) 405411.

[31] J. Jiang, K. Zhao, X. Xiao, L. Zhang, J. Am. Chem. Soc. 134 (2012) 4473-4476.

[32] N. Wu, J. Wang, D. N. Tafen, H. Wang, J. G. Zheng, J. P. Lewis, X. Liu, S. S. Leonard, A. Manivannan, J. Am. Chem. Soc. 132 (2010) 6679-6685.

[33] R. Li, F. Zhang, D. Wang, J. Yang, M. Li, J. Zhu, X. Zhou, H. Fan, C. Li, Nat. Commun. 4 (2013) 1432

[34] S. Bai, J. Jiang, Q. Zhang, Y. Xiong, Chem. Soc. Rev. 44 (2015) 2893-2939. 
[35] Z. B. Yu, Y. P. Xie, G. Liu, G. Q. Lu, X. L. Ma, H. M. Cheng, J. Mater. Chem. A 1 (2013) 2773-2776.

[36] T. Ohno, K. Sarukawa, M. Matsumura, New J. Chem. 26 (2002) 1167-1170.

[37] Y. Yin, A. P. Alivisatos, Nature 437 (2005) 664-670.

[38] M. A. Boles, D. Ling, T. Hyeon, D. V. Talapin, Nat. Mater. 15 (2016) 141-153.

[39] Y.-J. Chen, Y.-W. Chiang, M. H. Huang, ACS Appl. Mater. Interfaces 8 (2016) 19672-19679.

[40] C.-Y. Chu, M. H. Huang, J. Mater. Chem. A 5 (2017) 15116-15123.

[41] C. Wöll, Prog. Surf. Sci. 82 (2007) 55-120.

[42] E. S. Jang, J. H. Won, S.-J. Hwang, J. H. Choy, Adv. Mater. 18 (2006) 3309-3312.

[43] G. R. Li, T. Hu, G. L. Pan, T. Y. Yan, X. P. Gao, H. Y. Zhu, J. Phys. Chem. C 112 (2008) 11859-11864.

[44] A. McLaren, T. Valdes-Solis, G. Li, S. C. Tsang, J. Am. Chem. Soc. 131 (2009) 12540-12541.

[45] F. Liao, Y. Huang, J. Ge, W. Zheng, K. Tedsree, P. Collier, X. Hong, S. C. Tsang, Angew. Chem., Int. Ed. 50 (2011) 2162-2165.

[46] M. Behrens, F. Studt, I. Kasatkin, S. Kühl, M. Hävecker, F. Abild-Pedersen, S. Zander, F. Girgsdies, P. Kurr, B.-L. Kniep, M. Tovar, R. W. Fischer, J. K. Nørskov, R. Schlögl, Science 336 (2012) 893-897.

[47] Y. Qin, X. Wang, Z. L. Wang, Nature 451 (2008) 809-813.

[48] L. Wang, Y. Kang, X. Liu, S. Zhang, W. Huang, S. Wang, Sens. Actuators, B 162 (2012) 237-243.

[49] F. Cataldo, New Front. Chem. 23 (2014) 99-110.

[50] Y. Shiraishi, S. Kanazawa, Y. Kofuji, H. Sakamoto, S. Ichikawa, S. Tanaka, T. Hirai, Angew. Chem. Int. Ed. 53 (2014) 13454-13459.

[51] U. Diebold, L. V. Koplitz, O. Dulub, Appl. Surf. Sci. 237 (2004) 336-342.

[52] S. A. Ansari, M. M. Khan, S. Kalathil, A. Nisar, J. Lee, M. H. Cho, Nanoscale 5 (2013) 9238-9246.

[53] Y. Lv, W. Yao, X. Ma, C. Pan, R. Zong, Y. Zhu, Catal. Sci. Technol. 3 (2013) 3136-3146.

[54] F. Kayaci, S. Vempati, I. Donmez, N. Biyikli, T. Uyar, Nanoscale 6 (2014) 10224-10234.

[55] X. Zhang, J. Qin, Y. Xue, P. Yu, B. Zhang, L. Wang, R. Liu, Sci. Rep. 4 (2014) 4596.

[56] T. Andelman, Y. Gong, M. Polking, M. Yin, I. Kuskovsky, G. Neumark, S. O’Brien, J. Phys. Chem. B 109 (2005) 14314-14318.

[57] H. Zeng, G. Duan, Y. Li, S. Yang, X. Xu, W. Cai, Adv. Funct. Mater. 20 (2010) 561-572.

[58] K. M. Wong, S. M. Alay-e-Abbas, Y. Fang, A. Shaukat, Y. Lei, J. Appl. Phys. 114 (2013) 034901.

[59] Y.-K. Peng, Y. Fu, L. Zhang, I. F. Teixeira, L. Ye, H. He, S. C. E. Tsang, ChemCatChem 9 (2017) 155-160.

[60] Y. K. Peng, L. Ye, J. Qu, L. Zhang, Y. Fu, I. F. Teixeira, I. J. McPherson, H. He, S. C. E. Tsang, J. Am. Chem. Soc. 138 (2016) $2225-2234$.

[61] B. Yu, C. Zhu, F. Gan, Y. Huang, Mater. Lett. 33 (1998) 247-250.

[62] L. Jing, Z. Xu, J. Shang, X. Sun, W. Cai, H. Guo, Mater. Sci. Eng. A 332 (2002) 356-361.

[63] S. Polarz, J. Strunk, V. Ischenko, M. W. E. van den Berg, O. Hinrichsen, M. Muhler, M. Driess, Angew. Chem. Int. Ed. 45 (2006) 2965-2969.

[64] J. J. Schneider, R. C. Hoffmann, J. Engstler, S. Dilfer, A. Klyszcz, E. Erdem, P. Jakes, R. A. Eichel, J. Mater. Chem. 19 (2009) 1449-1457.

[65] V. Ischenko, S. Polarz, D. Grote, V. Stavarache, K. Fink, M. Driess, Adv. Funct. Mater. 15 (2005) 1945-1954.

[66] A. Corma, Chem. Rev. 95 (1995) 559-614. 
[67] A. Bhan, A. Allian, G. Sunley, D. Law, E. Iglesia, J. Am. Chem. Soc. 129 (2007) 4919-4924.

[68] A. Zheng, S.-J. Huang, S.-B. Liu, F. Deng, Phys. Chem. Chem. Phys. 13 (2011) 14889-14901.

[69] E. Carter, A. F. Carley, D. M. Murphy, ChemPhysChem 8 (2007) 113-123;

[70] E. Carter, A. F. Carley, D. M. Murphy, J. Phys. Chem. C 111 (2007) 10630-10638.

[71] W. Q. Fang, X.-Q. Gong, H. G. Yang, J. Phys. Chem. Lett. 2 (2011) 725-734.

[72] S. Liu, J. Yu, M. Jaroniec, Chem. Mater. 23 (2011) 4085-4093.

[73] M. V. Dozzi, E. Selli, Catalysts 3 (2013) 455-485.

[74] G. Liu, H. G. Yang, J. Pan, Y. Q. Yang, G. Q. Lu, H.-M. Cheng, Chem. Rev. 114 (2014) 9559-9612.

[75] A. Selloni, Nat. Mater. 7 (2008) 613-615.

[76] H. G. Yang, C. H. Sun, S. Z. Qiao, J. Zou, G. Liu, S. C. Smith, H. M. Cheng, G. Q. Lu, Nature 453 (2008) 638-642.

[77] X. Han, Q. Kuang, M. Jin, Z. Xie, L. Zheng, J. Am. Chem. Soc. 131 (2009) 3152-3153.

[78] H. G. Yang, G. Liu, S. Z. Qiao, C. H. Sun, Y. G. Jin, S. C. Smith, J. Zou, H. M. Cheng, G. Q. Lu, J. Am. Chem. Soc. 131 (2009) 4078-4083.

[79] S. Liu, J. Yu, M. Jaroniec, J. Am. Chem. Soc. 132 (2010) 11914-11916.

[80] J. Pan, G. Liu, G. Q. Lu, H.-M. Cheng, Angew. Chem. Int. Ed. 50 (2011) 2133-2137.

[81] T. R. Gordon, M. Cargnello, T. Paik, F. Mangolini, R. T. Weber, P. Fornasiero, C. B. Murray, J. Am. Chem. Soc. 134 (2012) 6751-6761.

[82] J. Yu, L. Qi, M. Jaroniec, J. Phys. Chem. C 114 (2010) 13118-13125.

[83] Q. Xiang, K. Lv, J. Yua, Appl Catal B. 95 (2010) 557-564.

[84] Y. Jiang, J. Scott, R. Amal, Appl Catal B. 126 (2012) 290-297.

[85] T. Tachikawa, S. Yamashita, T. Majima, J. Am. Chem. Soc. 133 (2011) 7197-7204.

[86] M. D’Arienzo, J. Carbajo, A. Bahamonde, M. Crippa, S. Polizzi, R. Scotti, L. Wahba, F. Morazzoni, J. Am. Chem. Soc. 133 (2011) 17652-17661.

[87] J. Yu, J. Low, W. Xiao, P. Zhou, M. Jaroniec, J. Am. Chem. Soc. 136 (2014) 8839-8842.

[88] X. Yu, B. Jeon, Y. K. Kim, ACS Catal. 5 (2015) 3316-3322.

[89] Y. Luan, L. Jing, Y. Xie, X. Sun, Y. Feng, H. Fu, ACS Catal. 3 (2013) 1378-1385.

[90] Q. Wu, M. Liu, Z. Wu, Y. Li, L. Piao, J. Phys. Chem. C 16 (2012) 26800-26804.

[91] F. Xiong, Y.-Y. Yu, Z. Wu, G. Sun, L. Ding, Y. Jin, X.-Q. Gong, W. Huang, Angew. Chem. Int. Ed. 55 (2016) 623-628.

[92] J. Yu, J. Fan, K. Lv, Nanoscale 2 (2010) 2144-2149.

[93] X. Wu, Z. Chen, G. Q. Lu, L. Wang, Adv. Funct. Mater. 21 (2011) 4167-4172.

[94] F. Hao, X. Wang, C. Zhou, X. Jiao, X. Li, J. Li, H. Lin, J. Phys. Chem. C 116 (2012) 19164-19172.

[95] L. Chu, Z. Qin, J. Yang, X. Li, Sci. Rep. 5 (2015) 12143.

[96] J. S. Chen, Y. L. Tan, C. M. Li, Y. L. Cheah, D. Luan, S. Madhavi, F. Y. C. Boey, L. A. Archer, X. W. Lou, J. Am. Chem. Soc. 132 (2010) 6124-6130.

[97] X.-L. Cheng, M. Hu, R. Huang, J.-S. Jiang, ACS Appl. Mater. Interfaces 6 (2014) 19176-19183.

[98] L. Atanda, S. Mukundan, A. Shrotri, Q. Ma, J. Beltramini, ChemCatChem 7 (2015) 781-790.

[99] L. Atanda, A. Shrotri, S. Mukundan, Q. Ma, M. Konarova, J. Beltramini, ChemSusChem 8 (2015) 2907-2916.

[100] X. C. Wang, J. C. Yu, P. Liu, X. X. Wang, W. Y. Su, X. Z. Fu, J. Photochem. Photobiol. A 179 (2006) 339-347. 
[101] H. Zhang, H. Yu, A. Zheng, S. Li, W. Shen, F. Deng, Environ. Sci. Technol. 42 (2008) 5316-5321.

[102] B. E. Hardin, H. J. Snaith, M. D. McGehee, Nat. Photonics 6 (2012) 162-169.

[103] H. Kusama, H. Orita, H. Sugihara, Langmuir 24 (2008) 4411-4419.

[104] Y.-K. Peng, Y. Hu, H.-L. Chou, Y. Fu, I. F. Teixeira, L. Zhang, H. He, S. C. E. Tsang, Nat. Commun. (2017) doi:10.1038/s41467-017-00619-z.

[105] S. Selçuk, A. Selloni, J. Phys. Chem. C 117 (2013) 6358-6362.

[106] W. Yuan, Y. Wang, H. Li, H. Wu, Z. Zhang, A. Selloni, C. Sun, Nano Lett. 16 (2016) 132-137.

[107] W. Yuan, H. Wu, H. Li, Z. Dai, Z. Zhang, C. Sun, Y. Wang, Chem. Mater. 29 (2017) 3189-3194.

[108] G. Liu, H. G. Yang, X. Wang, L. Cheng, H. Lu, L. Wang, G. Q. Lu, H.-M. Cheng, J. Phys. Chem. C 113 (2009) 21784-21788.

[109] W. Wei, N. Yaru, L. Chunhua, X. Zhongzi, RSC Adv. 2 (2012) 8286-8288.

[110] X. H. Yang, Z. Li, C. Sun, H. G. Yang, C. Li, Chem. Mater. 23 (2011) 3486-3494.

[111] K. Lv, Q. Xiang, J. Yua, Appl Catal B. 104 (2011) 275-281.

[112] H. Park, W. Choi, J. Phys. Chem. B 108 (2004) 4086-4093. 